\title{
Cuestionario de Sucesos Vitales (CSV): Estructura factorial, propiedades psicométricas y datos normativos
}

\author{
Bonifacio Sandín y Paloma Chorot \\ Universidad Nacional de Educación a Distancia (UNED), Madrid, España
}

Life Events Questionnaire (CSV): Factor structure, psychometric properties and normative data

\begin{abstract}
The aim of the present study was to validate the Life Events Questionnaire (Cuestionario de Sucesos Vitales, CSV). Two large and independent samples were used (age range 19 to 67 years). A protocol including the CSV, the PANAS Scales, the DSM5 Self-Rated Level 1 Cross-Cutting Symptom Measure-Adult, the Anxiety Scale (EA), the Depression Questionnaire (CD), and the Health Questionnaire (CS) was completed on two separate occasions (with a 6-month interval). Exploratory and confirmatory factor analyses revealed a 7-factor structure of the CSV, suggesting seven stress-relevant psychosocial domains. Scores on the questionnaire were predictive of elevated levels of psychopathology, anxiety, depression, and diseases assessed 6 months later, after controlling for the effect of negative and positive affectivity, age and gender. Evidence of reliability, normative data, and validity of the CSV is also reported. We conclude that the CSV provides a suitable measure for psychosocial stress assessment.
\end{abstract}

Keywods: Life Events Questionnaire; CSV; psychosocial stress; negative life events; psychopathology; anxiety; depression; disease.

Resumen: El objetivo del estudio fue validar el Cuestionario de Sucesos Vitales (CSV). Se utilizaron dos muestras amplias e independientes (rango de edad: 19-67 años). Se aplicó un protocolo en dos momentos temporales (separados por 6 meses) que incluía el CSV, las escalas PANAS de afecto positivo y negativo, el DSM-5 Self-Rated Level 1 Cross-Cutting Symptom Measure-Adult), la Escala de Ansiedad (EA), el Cuestionario de Depresión (CD), y el Cuestionario de Salud (CS). Los análisis factoriales exploratorios y confirmatorios revelaron una estructura de 7 factores que sugieren siete dimensiones psicosociales relevantes al estrés. El CSV predijo las puntuaciones en psicopatología, ansiedad, depresión y enfermedades, evaluadas 6 meses más tarde, tras controlar el efecto de la afectividad negativa y positiva, la edad y el género. También se proporciona evidencia de fiabilidad, datos normativos y validez del CSV. Se concluye que el CSV constituye una herramienta adecuada para la evaluación del estrés psicosocial.

Palabras clave: Cuestionario de Sucesos Vitales; CSV; estrés psicosocial; sucesos vitales negativos; psicopatología; ansiedad; depresión; enfermedad.a

\section{Introducción}

Aparte de la confusión (no sólo popular sino también científica) que suele existir en la interpretación sobre lo

Recibido 15 septiembre 2016; aceptado 11 diciembre 2016.

Correspondencia:

Bonifacio Sandín, Universidad Nacional de Educación a Distancia (UNED), Facultad de Psicología, Juan del Rosal 10, 28040 Madrid, España. Correo-e: bsandin@psi.uned.es

Paloma Chorot, Universidad Nacional de Educación a Distancia (UNED), Facultad de Psicología, Juan del Rosal 10, 28040 Madrid, España. Correo-e: pchorot@psi.uned.es que es el estrés, su conceptualización ha venido enfatizando diferentes enfoques o puntos de vista, entre los que se incluyen la consideración del estrés como una respuesta (p.ej., activación del sistema hipotálamo-hipófiso-corticosuprarrenal), como un estímulo (p.ej., un suceso vital), o como un proceso de interacción entre el estímulo y la respuesta (p.ej., aproximaciones mediacionales o interaccionales del estrés) (véase Sandín, 2009). Los enfoques del estrés basados en el estímulo han tenido gran repercusión sobre la psicología clínica en las últimas décadas, siendo el más influyente el conocido como «enfoque psicosocial del estrés», o enfoque sobre 
los sucesos vitales, cambios vitales, o estrés reciente (Sandín, 2008). Es importante subrayar que este enfoque sólo cubre una faceta del estrés psicosocial, ya que no incluye otras formas relevantes de estrés psicosocial, tales como el estrés diario (Santed, Sandín, y Chorot, 1996, 1998; Santed, Sandín, Chorot, y Olmedo, 2000), el estrés crónico (Sandín, 2003, 2008), o el estrés asociado a eventos traumáticos (Echeburúa, 2009).

El enfoque basado en los sucesos vitales se inició a partir del modelo biopsicosocial desarrollado por el grupo de Holmes (p.ej., Holmes y David, 1989) desde los años sesenta. Este autor fundamentó su teoría en el hecho de que las personas parecen enfermar por factores que no son estrictamente biológicos o físicos (virus, bacterias, etc.), tales como los factores sociales o contextuales. El modelo biopsicosocial de este autor enfatizó el papel etiológico de los factores sociales y psicológicos en la etiopatogénesis de las enfermedades, oponiéndose así a la orientación médica biologicista propia de la época. Partiendo de esta idea, Holmes desarrolló el concepto de estrés psicosocial basándose en la idea de suceso vital como «cambio vital». Los sucesos vitales fueron definidos como eventos sociales que requieren algún tipo de cambio respecto al ajuste habitual del individuo, provocando en el organismo la necesidad de algún tipo de reajuste psicobiológico; por ello, el suceso vital puede identificarse con el concepto de cambio vital (a mayor cambio, por ejemplo por la suma de varios sucesos vitales, mayor probabilidad de enfermar).

Un aspecto esencial de este nuevo enfoque fue la posibilidad de evaluar el estrés mediante la cuantificación del cambio o reajuste exigido al individuo a través de los sucesos vitales, lo cual llevó a cabo a través de su conocida escala de reajuste social (Social Readjustment Rating Scale, SRRS; Holmes y Rahe, 1967). La SRRS constituye el primer método escalado para cuantificar el estrés, y evalúa los sucesos vitales como experiencias objetivas que generan una necesidad de reajuste sustancial en el individuo. Holmes y Rahe asignaron a cada ítem de la escala (43 sucesos) una puntuación de estrés en función del nivel de reajuste exigido por el suceso, variando entre 100 (muerte del cónyuge) y 11 (violaciones menores de la ley). Aunque estos sucesos vitales pueden ser traumáticos, generalmente consisten en eventos más o menos habituales o normativos dentro de la población, tales como el matrimonio, el divorcio o separación, la jubilación, la pérdida de trabajo, un accidente de tráfico, la pérdida de un amigo íntimo, etc. Esta forma de interpretar y cuantificar el estrés posibilita estudiar la relación entre el estrés y la salud examinando el efecto simultáneo de múltiples sucesos vitales experimentados por el individuo. Así mismo, se asumía que la vulnerabilidad atribuida a los sucesos vitales actuaba de forma no específica sobre cualquier tipo de enfermedad o trastorno mental (Holmes y David, 1989; Sandín, 2008). Por otra parte, para evitar problemas de fiabilidad debido a posibles sesgos de memoria, esta forma de evaluación retrospectiva no suele circunscribirse a más de los 12 meses previos (i.e., sucesos vitales ocurridos durante el último año), por lo que habitualmente esta faceta del estrés psicosocial suele entenderse como una forma de estrés reciente.

Este novedoso enfoque, no sólo fue seguido por el propio grupo de Holmes, sino también por otros muchos investigadores que, bien aplicando la propia SRRS, o bien desarrollando otros instrumentos similares o derivados de ella, han generado un cuerpo de evidencia amplio y significativo sobre la asociación e implicación del estrés psicosocial reciente en el desarrollo y/o patogenia de las principales enfermedades y trastornos mentales. Aunque un análisis sobre esta cuestión sobrepasa los objetivos y límites del presente estudio, los sucesos vitales se han asociado a la salud física en general (Holmes y David, 1989; Sandín, 1989, 1993, 2008), la sintomatología somática (Sandín, Chorot, Santed, y Valiente, 2002), y la mayor parte de las perturbaciones y enfermedades físicas, incluidas las enfermedades cardiovasculares (Bernston, Patel, y Steward, 2017; Chorot y Sandín, 1994; Sandín, Chorot, Santed, y Jiménez, 2009), el cáncer (Chorot y Sandín, 1994; Heikkinen et al., 2017; Sandín et al., 2009), los trastornos gastrointestinales (Tobón, Sandín, y Vinaccia, 2006; Tobón, Vinaccia, y Sandín, 2003, 2004; Vinaccia, Tobón, Martínez, y Sandín, 2001; Vinaccia, Tobón, Sandín, y Martínez, 2001), la diabetes y en general las enfermedades autoinmunes y la resistencia a la enfermedad (Holmes y David, 1989; Porcelli et al., 2016; Sandín et al., 2009).

De modo similar, existe amplia evidencia sobre la asociación entre la experiencia previa de sucesos vitales negativos y la perturbación de la salud mental (véase Miller, 1997). A este respecto, y únicamente a modo de ejemplo, merece destacarse la implicación del estrés por sucesos vitales en la vulnerabilidad, génesis y/o agravación de diferentes tipos y manifestaciones de los trastornos mentales, incluidos los trastornos de ansiedad (Phillips, Carroll, y Der, 2015; Sandín y Chorot, 1993; Sandín, Chorot, Santed, y Valiente, 2004), la depresión (Hammen, 2005; Phillips et al., 2015; Sandín et al., 2004), los trastornos psicóticos (Mansueto y Faravelli, 2017), el riesgo de suicidio (Xavier, Cunha, y Pinto-Gouveia, 2015; Kokoulina y Fernández, 2014; Liu y Miller, 2014; Sandín, Chorot, Santed, Valiente, y Joiner, 1998), el abuso de sustancias (Krenek y Maisto, 2013), el burnout (Ballester-Arnal et al., 2016; Esteras, Chorot, 
y Sandín, 2014), el trastorno de pánico (Sandín, Rodero, Santed, y García-Campayo, 2006), y en general el nivel de psicopatología (Clements y Turping, 1996; Miller, 1997; Santandreu y Ferrer, 2014; Valiente, Sandín, Chorot, Santed, y González de Rivera, 1996).

A pesar de la enorme influencia de la SRRS, desde el principio fue criticada por presentar diversos problemas metodológicos (véase Sandín, 2008; Sandín y Chorot, 1996), entre los cuales se incluyen los problemas de validez de contenido (por el limitado muestreo de sucesos vitales) y la no evaluación del impacto diferencial (distress) producido por los sucesos vitales (i.e., un mismo suceso vital puede ser muy estresante para un individuo pero mucho menos para otro). Con objeto de solucionar estos problemas, a finales de los años ochenta desarrollamos el Cuestionario de Sucesos Vitales (CSV), mediante el cual pretendíamos disponer de un instrumento para evaluar el estrés reciente aplicable a la población general de habla española que, por una parte tuviese en cuenta las distintas dimensiones psicosociales asociadas a los sucesos vitales y, por otra, que posibilitase evaluar los sucesos vitales según la percepción que el propio individuo tiene de cada suceso particular experimentado.

Un primer objetivo del presente estudio consistió en examinar la prevalencia de los sucesos vitales, así como el nivel de estrés producido por los distintos sucesos incluidos en el cuestionario. A través de un segundo objetivo deseábamos examinar la estructura factorial del CSV, esperando que dicha estructura refleje dimensiones específicas que puedan representar áreas relevantes de estrés psicosocial. Mediante un tercer objetivo hemos pretendido obtener datos normativos sobre el CSV, incluyendo las puntuaciones medias en las diferentes escalas, desviaciones típicas, y puntos de corte basados en percentiles. Finalmente, examinamos la fiabilidad (consistencia interna) y validez (convergente, discriminante y predictiva) del cuestionario.

\section{Método}

\section{Participantes}

El número total de participantes en el estudio consistió en 1.871 estudiantes de los dos primeros cursos de psicología de la Universidad Nacional de Educación a Distancia. La muestra total incluía participantes de todas las regiones de España; algunos residían en el extranjero, fundamentalmente en países hispanoamericanos (México, Argentina, Venezuela y Perú) (véase la Tabla 1). Utilizamos dos muestras independientes. La Muestra 1 consistió en 867 participantes (edad: $M=33.2$ años, rango = 19-67 años), de los cuales 623 eran mujeres (71.9\%) y
244 eran varones (28.1\%). La Muestra 2 consistió en 984 participantes (edad: $M=32.4$ años, rango $=19-67$ años), de los cuales 769 eran mujeres (78.2\%) y 215 eran varones (21.8\%); parte de esta muestra (la Submuestra 2, $n=$ 739) cumplimentó el protocolo también en una segunda ocasión, 6 meses más tarde). La mayor parte de los participantes eran de raza blanca (aproximadamente el 85\%), siendo los restantes hispanos y de raza negra. Respecto al estado civil, el 59.8\% de los participantes eran solteros, el $33.1 \%$ estaban casados o vivían en pareja de hecho, y el $7.1 \%$ estaban separados, divorciados o viudos.

Tabla 1. Distribución de la muestra a través de las distintas regiones de España $(N=1.851)$

\begin{tabular}{lcc}
\hline \multicolumn{1}{c}{ Región } & Frecuencia & Porcentaje \\
\hline Andalucía & 326 & 17.6 \\
Aragón & 133 & 7.2 \\
Asturias & 54 & 2.9 \\
Baleares & 36 & 1.9 \\
Canarias & 76 & 4.1 \\
Cantabria & 41 & 2.2 \\
Castilla La Mancha & 125 & 6.8 \\
Castilla León & 100 & 5.4 \\
Cataluña & 98 & 5.3 \\
Extremadura & 34 & 1.8 \\
Galicia & 101 & 5.5 \\
La Rioja & 14 & 0.8 \\
Madrid & 403 & 21.8 \\
Murcia & 46 & 2.5 \\
Navarra & 44 & 2.4 \\
País Vasco & 57 & 3.1 \\
Valencia & 137 & 7.4 \\
Ceuta Melilla & 10 & 0.5 \\
Extranjero & 16 & 0.9 \\
\hline
\end{tabular}

\section{Instrumentos}

Cuestionario de Sucesos Vitales (CSV; Sandín y Chorot, 1987) (véase Sandín y Chorot, 2008; Sandín, Valiente y Chorot, 2008). Consta de una lista de 60 sucesos vitales relacionados con diversas áreas psicosociales, entre las que se incluyen el trabajo, el ámbito académico, la salud, las relaciones de pareja, la familia e hijos, las relaciones sociales, la residencia, y los aspectos económicos y legales. El sujeto contesta a cada suceso vital experimentado durante el último año indicando (a) el impacto producido por el suceso (grado de estrés o malestar percibido) según una escala que varía entre 1(nada o muy poco estrés) y 4 (muchísimo estrés); debe señalar el 0 en el caso de que no haya experimentado el suceso durante el último año. Sobre las distintas formas del cuestionario se han obtenido datos que sugieren niveles aceptables de fiabilidad y buenos niveles de validez con- 
vergente, discriminante y predictiva (Sandín, 2008). Aparte de la valoración del nivel de estrés o malestar percibido en relación con cada suceso, el CSV permite cuantificar la valencia (i.e., si el suceso ha sido valorado como algo positivo o negativo) y la predecibilidad (i.e., si el suceso fue algo esperado o sucedió de forma inesperada para el individuo) (los datos que presentamos en este estudio se refieren únicamente a la evaluación del nivel de estrés o malestar percibido). En el Anexo I se incluye el CSV actual en formato de aplicación, con indicación de las diferentes áreas psicosociales que pueden evaluarse a través del mismo.

Escalas de Afecto Positivo y Negativo [Positive and Negative Affect Schedule, PANAS] (Watson, Clark y Tellegen, 1988). Se aplicó la versión española validada por Sandín et al. (1999). Consta de 20 ítems, 10 referidos al afecto positivo y 10 al afecto negativo. Cada ítem se puntúa según una escala de intensidad de 1 («nada o casi nada») a 5 («muchísimo»). Las propiedades psicométricas de esta versión son excelentes. En el presente estudio, los coeficientes alfa de Cronbach obtenidos con la muestra total de participantes fueron de .91 y .90 para las escalas de afecto positivo y negativo, respectivamente.

Escala de Ansiedad (EA; Sandín, Chorot y Valiente, 2011). La EA es una escala breve (10 ítems) de autoinforme desarrollada recientemente por nuestro grupo para (a) obtener una evaluación de la ansiedad de forma rápida y libre de contaminación de otros constructos cercanos como la depresión o el afecto negativo, y (b) no maximizar o reducirse a los síntomas de activación fisiológica. El participante contesta indicando la frecuencia con que ha experimentado durante las últimas semanas lo indicado en cada ítem, según una escala que varía entre 0 («nunca o casi nunca») y 4 («muchas veces o casi siempre»). El coeficiente alfa de Cronbach obtenido en el presente estudio con la Muestra 2 fue de .94. La correlación test-retest (6 meses) fue de .70 (Submuestra 2).

Cuestionario de Depresión (CD; Sandín y Valiente, 1998) (véase Sandín y Chorot, 2008; Sandín, Valiente y Chorot, 2008). El CD es un cuestionario de autoinforme de 16 ítems diseñado para evaluar la depresión (a) en un tiempo breve, (b) sin solaparse con otros constructos cercanos como ansiedad o afectividad negativa, y (c) sobre la base de los síntomas del trastorno depresivo mayor y del trastorno distímico; el cuestionario se ajusta a los criterios actuales del DSM-5 (American Psychiatric Association, 2013a). El participante contesta indicando la frecuencia con que ha experimentado durante las últimas semanas lo indicado en cada ítem, según una escala que varía entre 0 («nunca o pocas veces») y 4 («casi siempre»). El coeficiente alfa de Cronbach obtenido en el presente estudio con la Muestra 2 fue de .92. La correlación test-retest (6 meses) fue de .73 (Submuestra 2).

Cuestionario Transversal de Síntomas DSM-5 [DSM-5 Self-Rated Level 1 Cross-Cutting Symtom Measure-Adult] (American Psychiatric Association, 2013b). El DSM-5 Transversal es un cuestionario de autoinforme de 23 ítems desarrollado por la APA como material complementario al DSM-5 para establecer un screening de los síntomas psicopatológicos. Aparte de la información específica sobre un posible screening psicopatológico, proporciona una medida general de síntomas psicopatológicos. El cuestionario se cumplimenta contestando a cada ítem según una escala compuesta de intensidad y frecuencia que varía entre 0 (ninguna/nada en absoluto) y 4 (grave/casi cada día), referido a las dos últimas semanas. Se utilizó la versión española de Sandín, Chorot y Valiente (2013). El cuestionario fue inicialmente traducido al español por estos autores y, posteriormente, se efectuó la traducción inversa por un psicólogo clínico con lengua nativa inglesa, consensuándose la versión española final. En el actual estudio obtuvimos con la Muestra 2 un coeficiente alfa de fiabilidad de .92. La correlación test-retest (6 meses) fue de .66 (Submuestra 2).

Cuestionario de Salud (CS). Este cuestionario fue diseñado para la investigación relacionada con el presente estudio y está constituido por 8 ítems que los participantes contestan según una escala de frecuencia (entre 0 y 4). Los ítems se refieren a conductas relacionadas con la salud (ejercicio físico, tabaco, y alcohol; 3 ítems) y enfermedades (enfermedades ocurridas y atenciones médicas; 4 ítems), además de un ítem sobre percepción de la propia salud. Se aplicó referido al último año (Muestra 2) y a los 6 últimos meses (Submuestra 2). Para el presente estudio únicamente se utilizaron los 4 ítems referidos a enfermedades (coeficiente alfa de Cronbach $=.60$, obtenido a partir de la Muestra 2).

\section{Procedimiento}

Los participantes se obtuvieron a través de anuncios en la web de la Universidad Nacional de Educación a Distancia durante un periodo de un mes aproximadamente. Se les invitó a participar voluntariamente en el estudio, indicándoles que su tarea consistiría en contestar a varios cuestionarios relacionados con diferentes reacciones emocionales que suele experimentar la gente. Se indicó, así mismo, (a) que su colaboración podía pararla en cualquier momento si ésta no era de su agrado excluyéndose así del estudio, y (b) que los participantes que finalizaran el protocolo serían recompensados con un crédito de prácticas. Todos los participantes 
firmaron vía internet el consentimiento informado. No fue necesario eliminar ningún protocolo por errores $u$ omisiones relacionados con la aplicación ya que la plataforma online utilizada impedía este tipo de problemas comunes en las aplicaciones mediante papel y lápiz. Las Muestras 1 y 2 cumplimentaron el protocolo de forma independiente, con una separación entre ambas de tres años. La Muestra 2 (Submuestra 2) cumplimentó el protocolo también en una segunda ocasión 6 meses más tarde.

\section{Análisis estadístico de los datos}

Previamente se calcularon los datos descriptivos referidos a la frecuencia e intensidad de los ítems del CSV, con el fin de examinar los sucesos más frecuentes y el nivel de intensidad del estrés percibido asociado a cada ítem. Posteriormente, con objeto de aislar los componentes psicosociales básicos, aplicamos análisis de componentes principales (ACP) a los 60 ítems del CSV. Este método de extracción es ideal para llevar a cabo una selección de un conjunto de elementos relevantes sin modificar los componentes básicos asociados a los mismos (Brown, 2006; Tabachnick y Fidell, 2007). La determinación del número de componentes o factores se llevó a cabo aplicando el test de scree (análisis visual de los autovalores; Catell, 1966) y el método paralelo (Horn, 1965) (este último método se aplicó comparando los valores de los autovalores de los factores con el percentil 95 de los autovalores que se producen al azar). Aplicamos rotación oblicua, método promax. Todos los ACP se efectuaron a partir de los datos procedentes de la Muestra 1. Previo a la realización de los análisis se calculó la prueba de esfericidad de Bartlett y el coeficiente de Kaiser-Meyer-Olkin, con objeto de asegurarnos de la adecuación de la muestra.

En una segunda fase llevamos a cabo análisis factoriales confirmatorios (AFC) sobre los elementos preseleccionados a través del ACP basándonos en los datos de la Muestra 2. Mediante los AFC deseábamos validar la estructura factorial previa aislada a través de ACP. La definición del modelo se efectuó del siguiente modo. En la construcción del modelo, cada grupo de ítems del cuestionario se asoció a una única variable latente (factor psicosocial) de acuerdo con las especificaciones sugeridas por el ACP. Las varianzas de los términos de error fueron especificadas como parámetros libres. Como norma no se permitió la covariación entre los residuos. La varianza de las variables latentes se fijó en 1.00 con objeto de identificar el modelo de medida. Los análisis se basaron en las correlaciones policóricas y se utilizó el método de estimación ML-robusto, el cual pro- porciona estadísticos robustos a partir de la prueba escalada de $\chi^{2}$ de Satorra-Bentler (S-B $\chi^{2}$ ) y errores estándar robustos, corrigiendo los errores debidos a una posible violación del principio de normalidad. La aplicación de este método significa que los valores calculados son válidos incluso aunque se violase la asunción de normalidad en el método de estimación. Siguiendo a Brown (2006), utilizamos índices de bondad de ajuste de diversas clases (absolutos, de parsimonia y comparativos): (1) $\mathrm{S}-\mathrm{B} \chi^{2}$ ( $\chi^{2}$ de Satorra-Bentler), (2) S-B $\chi^{2}$ dividido por los grados de libertad (cocientes $\leq 5.0$ indican buen ajuste; a menor índice mejor ajuste), (3) CFI (robust comparative fit index), (4) SRMR (standardized root mean-square residual), y (6) RMSEA (root mean square error of approximation). Se considera un ajuste aceptable si los valores de CFI $\geq 0.90$, SRMR $\leq 0.08$, y RMSEA $\leq 0.06$ (Byrne, 2006; Hu y Bentler, 1999). Estos análisis se llevaron a cabo a partir de la Muestra 2.

Finalmente, se calcularon las puntuaciones normativas $(M$ y $D T)$ para las diferentes escalas del CSV y otras variables del estudio aplicando análisis de varianza univariados y calculando el tamaño del efecto para examinar las diferencias en función del género. La validez convergente y discriminante del CSV se obtuvo mediante el cálculo de correlaciones, y su validez predictiva a través de análisis de regresión múltiple. También calculamos los datos normativos del cuestionario estableciendo los puntos corte para niveles de percentiles. Todos los análisis del estudio se llevaron a cabo utilizando los programas estadísticos SPSS.24 y EQS 6.3.

\section{Resultados}

\section{Frecuencia de los sucesos vitales}

Con objeto de obtener evidencia sobre la prevalencia anual de los sucesos vitales en la población estudiada examinamos la frecuencia y el porcentaje correspondientes a cada uno de los 60 sucesos representados en el CSV, cuyos resultados se indican en la Tabla 2. Debe tenerse en cuenta que dos de los sucesos más prevalentes (Ítems 10 y 41) están determinados por el tipo de muestra utilizada, ya que ésta corresponde a población académica universitaria. Si consideramos el $20 \%$ como punto de corte de una frecuencia elevada, se constata que el $40 \%$ (24 sucesos) de todos los acontecimientos descritos en el CSV poseen una prevalencia alta. Con el fin de comparar la distribución de las frecuencias entre los grupos de mujeres y varones aplicamos tablas de contingencia (se calculó el estadístico de $\chi^{2}$ ) a cada uno de los ítems del CSV, obteniendo diferencias significativas entre ambos grupos únicamente para 12 de los sucesos vi- 
tales del cuestionario (véase la Tabla 2). Finalmente deseábamos comprobar si los perfiles de las mujeres y los varones para los sucesos más comunes (24 sucesos más frecuentes; i.e., sucesos experimentados por al menos el $20 \%$ de los participantes) eran similares, para lo cual calculamos el coeficiente rho de Spearman (orden de rangos), obteniéndose una correlación muy elevada (rho $=.94, p<.001$ ), lo que indica que existe una elevada similitud en el orden de frecuencia de los sucesos vitales experimentados por los hombres y las mujeres.

\section{Los sucesos vitales más estresantes}

Un paso siguiente en nuestro análisis sobre los sucesos vitales vividos durante el último año consistió en examinar su nivel de impacto, o nivel de estrés o malestar psicológico (distrés) experimentado por los participantes. En la Tabla 3 se indican los niveles de estrés percibido correspondiente a todos y cada uno de los sucesos vitales del CSV experimentados. Los sucesos los hemos diferenciado en función del grado de estrés experimentado por los sujetos, partiendo del rango de valoración de la escala, i.e., entre ninguno o muy poco estrés (1) y muchísimo estrés (4). Tal y como indicamos en la tabla, aunque existen algunas diferencias entre hombres y mujeres, sólo 8 sucesos fueron percibidos con elevado nivel de estrés (puntuaciones por encima de 2.50), correspondiendo todos ellos a pérdidas de seres queridos y a relaciones de pareja (ruptura de noviazgo, divorcio o separación, etc.). Un segundo grupo corresponde a 23 sucesos vitales que fueron percibidos con nivel de estrés moderado-alto (i.e., entre 2.00 y 2.50 ), e incluye diversas categorías de acontecimientos (de tipo legal, económico, familiar, laboral, etc.). Finalmente, un tercer grupo (resto de sucesos) corresponde a los acontecimientos percibidos con bajo nivel de estrés (i.e., puntuaciones menores de 2.00).

Para comparar el nivel de estrés percibido por los grupos de participantes (mujeres vs. varones) en relación con cada suceso experimentado aplicamos la prueba de $t$ de Student referida a cada uno de los ítems del $\mathrm{CSV}$, encontrando diferencias estadísticamente significativas en 23 de los ítems. Las diferencias más marcadas correspondían a sucesos relacionados con la salud y las relaciones de pareja (véase la Tabla 3). Para examinar la correspondencia en el orden de gravedad de los sucesos experimentados por ambos grupos calculamos la correlación rho de Spearman basada en rangos (orden de intensidad) a los 31 sucesos con nivel de estrés significativo (entre los niveles de estrés alto y moderado) (se suprimen los ítems específicos de grupo de género). Los resultados de este análisis indicaron un perfil sólo relati- vamente similar $(r h o=.64, p<.001)$. Como puede apreciarse en la Tabla 3 , aunque los sucesos más estresantes lo son para ambos grupos, los sucesos con niveles de estrés moderado presentan mayor variabilidad en el orden de intensidad de estrés percibido al comparar entre ambos grupos.

\section{Análisis factorial exploratorio}

Para aislar las posibles áreas psicosociales o dimensiones del CSV y seleccionar los ítems relevantes a las mismas aplicamos análisis de componentes principales basándonos en la Muestra 1. Previo a los análisis factoriales se calculó la prueba de adecuación de la muestra de Kaiser-Meyer-Olkin (= .728) y la prueba de esfericidad de Bartlett $\left[\chi^{2}=5539.9(780), p=0.000\right]$. El conjunto de items del CSV fue sometido a análisis de componentes principales, obteniéndose 14 factores con autovalores superiores a la unidad. No obstante, los criterios de estructura simple y las pruebas de scree y paralelo sugerían 7 factores fiables. Basándonos en este análisis, se llevó a cabo un proceso de selección de ítems, eliminándose los que no saturaban de forma relevante en algunos de los 7 primeros factores, los items hiperplanos y los items con transaturaciones, quedando reducida la estructura factorial a 42 ítems, los cuales explicaban el $40 \%$ de la varianza (los autovalores correspondientes a estos 7 factores variaron entre 4.4 y 1.5). Los pesos factoriales, así como la descripción de los factores se indican en la Tabla 4.

Como puede apreciarse en dicha tabla, los siete factores aislados corresponden a siete áreas relevantes de estrés psicosocial. El primer factor (7 elementos), que denominamos «Trabajo», aparecía configurado por ítems relacionados estrictamente con cuestiones de tipo laboral. El segundo factor (denominado «Relaciones de pareja», 8 elementos) aglutina ítems relacionados con problemas de pareja, entre los que se incluye el divorcio o separación (este ítem también saturaba en el factor 7). El tercer factor («Vivienda/Legal/Social», 10 ítems) era un factor más genérico que los dos anteriores, ya que se configuraba con sucesos que hacían alusión a situaciones de amenaza/peligro/daño referidas a la vivienda (p.ej., hacer reformas en casa), cuestiones de tipo legal (p.ej., sufrir algún tipo de robo), y social en general (p.ej., problemas con los vecinos). El cuarto factor («Pérdida laboral/ingresos», 5 elementos) se refiere a un ámbito psicosocial caracterizado por la pérdida de condiciones laborales y/o ingresos económicos (p.ej., perder el trabajo). El quinto factor («Salud familia», 3 ítems) se refiere específicamente a problemas graves de salud de familiares cercanos. El sexto factor («Salud 
Tabla 2. Porcentaje de individuos que experimentaron cada suceso vital durante el último año (Muestras 1 y 2, $N=1.851$ )

\begin{tabular}{|c|c|c|c|}
\hline Número de ítem del CSV & $\begin{array}{c}\text { Total } \\
(N=1.851)\end{array}$ & $\begin{array}{c}\text { Mujeres } \\
(N=1.392)\end{array}$ & $\begin{array}{l}\text { Hombres } \\
(N=459)\end{array}$ \\
\hline 10. Exámenes importantes & $95.7 \%$ & $95.6 \%$ & $95.9 \%$ \\
\hline 32. Periodo de fiestas ${ }^{a}$ & $91.0 \%$ & $91.5 \%$ & $89.5 \%$ \\
\hline 41. Suspender exámenes & $71.7 \%$ & $73.1 \% *$ & $67.8 \%$ \\
\hline 3. Problemas con colegas o compañeros de trabajo & $62.3 \%$ & $60.6 \%$ & $67.8 \% * *$ \\
\hline 35. Cambio en las costumbres personales (salir, vestir, etc.) ${ }^{\text {b }}$ & $56.6 \%$ & $57.3 \%$ & $54.2 \%$ \\
\hline 11. Reducción sustancial en los ingresos & $54.1 \%$ & $55.3 \%$ & $50.3 \%$ \\
\hline 45. Enfermedad de un familiar & $50.2 \%$ & $51.2 \%$ & $47.3 \%$ \\
\hline 52. Hospitalización de algún familiar & $44.7 \%$ & $44.6 \%$ & $45.1 \%$ \\
\hline 59. Discusión especialmente grave con su cónyuge o pareja & $44.5 \%$ & $46.3 \% * *$ & $39.0 \%$ \\
\hline 27. Problemas con vecinos o con familiares que no viven en la casa familiar & $42.5 \%$ & $42.6 \%$ & $42.3 \%$ \\
\hline 57. Éxito personal importante & $40.7 \%$ & $41.0 \%$ & $39.7 \%$ \\
\hline 43. Enamorarse o iniciar una amistad íntima y profunda & $39.9 \%$ & $41.0 \%$ & $36.6 \%$ \\
\hline 25. Problema legal menor (multa, etc.) & $34.1 \%$ & $31.0 \%$ & $43.6 \% * * *$ \\
\hline 47. Cambio de horario o de las condiciones del trabajo actual & $32.8 \%$ & $32.5 \%$ & $33.8 \%$ \\
\hline 12. Reformas en la casa & $32.4 \%$ & $31.5 \%$ & $35.3 \%$ \\
\hline 56. Problemas con superiores en el trabajo & $27.4 \%$ & $26.2 \%$ & $31.2 \% *$ \\
\hline 1. Quedarse sin trabajo & $27.3 \%$ & $29.2 \% * *$ & $21.4 \%$ \\
\hline 26. Cambio de casa o tener que buscar una nueva vivienda & $25.2 \%$ & $26.2 \%$ & $22.0 \%$ \\
\hline 19. Ruptura de noviazgo o relación similar & $24.9 \%$ & $26.2 \% *$ & $20.7 \%$ \\
\hline 2. Enfermedad prolongada que requiere tratamiento & $23.1 \%$ & $23.3 \%$ & $22.4 \%$ \\
\hline 30. Nuevo empleo laboral & $22.0 \%$ & $23.0 \%$ & $19.0 \%$ \\
\hline 18. Cambio de lugar de trabajo & $21.0 \%$ & $21.0 \%$ & $21.1 \%$ \\
\hline 36. Enfermedad o accidente que requiere guardar cama & $20.9 \%$ & $20.1 \%$ & $23.1 \%$ \\
\hline 48. Vivir temporalmente fuera del hogar & $19.9 \%$ & $19.8 \%$ & $20.3 \%$ \\
\hline 54. Pérdidas económicas importantes no asociadas al trabajo & $18.0 \%$ & $17.0 \%$ & $20.9 \%$ \\
\hline 31. Muerte de algún familiar cercano (padre, hermano, etc.) & $17.9 \%$ & $18.5 \%$ & $16.1 \%$ \\
\hline 6. Problemas con los hijos (no poder verlos por separación, algún problema grave en el colegio, etc.) & $17.2 \%$ & $16.7 \%$ & $18.5 \%$ \\
\hline 55. Cambio de centro de estudios & $16.8 \%$ & $17.5 \%$ & $14.8 \%$ \\
\hline 37. Finalización de estudios & $15.3 \%$ & $15.2 \%$ & $15.5 \%$ \\
\hline 49. Reconciliación matrimonial o de pareja & $14.9 \%$ & $15.7 \%$ & $12.6 \%$ \\
\hline 33. Préstamo o hipoteca de más de $100.000 €$ & $13.6 \%$ & $13.4 \%$ & $13.9 \%$ \\
\hline 34. Sufrir robo & $12.7 \%$ & $13.2 \%$ & $11.3 \%$ \\
\hline 40. Lesión física importante & $12.3 \%$ & $11.9 \%$ & $13.5 \%$ \\
\hline 5. Matrimonio & $10.9 \%$ & $10.7 \%$ & $11.5 \%$ \\
\hline 38. Accidente de tráfico & $9.9 \%$ & $9.8 \%$ & $10.5 \%$ \\
\hline 58. El cónyuge comienza a trabajar fuera de casa & $9.9 \%$ & $10.5 \%$ & $8.1 \%$ \\
\hline 4. Retiro laboral (jubilarse) & $9.7 \%$ & $10.6 \% *$ & $7.0 \%$ \\
\hline 15. Ascenso en el trabajo & $9.3 \%$ & $8.6 \%$ & $11.3 \%$ \\
\hline 39. Repentino y serio deterioro de la audición o visión & $8.9 \%$ & $9.0 \%$ & $8.7 \%$ \\
\hline 22. Relaciones sexuales fuera del matrimonio & $7.7 \%$ & $7.2 \%$ & $9.4 \%$ \\
\hline 20. Muerte de un amigo íntimo * & $7.2 \%$ & $6.9 \%$ & $8.3 \%$ \\
\hline 17. Comprar una casa & $6.9 \%$ & $6.6 \%$ & $7.6 \%$ \\
\hline 51. Fracaso en algún negocio importante & $6.9 \%$ & $5.9 \%$ & $10.0 \% * *$ \\
\hline 23. Hijos lejos del hogar & $5.9 \%$ & $5.5 \%$ & $7.0 \%$ \\
\hline 14. Problemas legales relacionados con el alcohol o las drogas & $5.8 \%$ & $4.5 \%$ & $9.8 \% * * *$ \\
\hline 50. Separación de los padres & $5.6 \%$ & $5.7 \%$ & $5.0 \%$ \\
\hline 8. Divorcio o separación & $5.0 \%$ & $5.5 \%$ & $3.7 \%$ \\
\hline 53. Sufrir asalto o situación de violencia física & $4.9 \%$ & $4.5 \%$ & $6.1 \%$ \\
\hline 46. Ruptura de relaciones sexuales fuera del matrimonio & $4.5 \%$ & $4.0 \%$ & $6.1 \%$ \\
\hline 9. Problema legal grave (con o sin cárcel) & $4.4 \%$ & $3.7 \%$ & $6.5 \% * *$ \\
\hline 16. Sufrir malos tratos de su pareja & $4.4 \%$ & $4.4 \%$ & $4.6 \%$ \\
\hline 42. Quedarse embarazada & $3.8 \%$ & $5.0 \%$ & - \\
\hline 7. Nacimiento de un hijo & $3.6 \%$ & $2.9 \%$ & $5.7 \% * *$ \\
\hline 24. Complicación legal en una pelea & $2.5 \%$ & $2.3 \%$ & $3.1 \%$ \\
\hline 28. Abortar & $1.5 \%$ & $1.9 \%$ & - \\
\hline 44. Embarazo de su mujer o pareja & $1.5 \%$ & - & $5.2 \%$ \\
\hline 13. Muerte del cónyuge & $0.7 \%$ & $0.6 \%$ & $1.1 \%$ \\
\hline 60. Alquilar una vivienda ${ }^{c}$ & $0.5 \%$ & $0.5 \%$ & $0.7 \%$ \\
\hline 29. Aborto de su mujer o pareja & $0.4 \%$ & - & $1.7 \%$ \\
\hline 21. Muerte de un hijo & $0.3 \%$ & $0.4 \%$ & $0.2 \%$ \\
\hline
\end{tabular}

Nota. ${ }^{a}$ Este ítem ha sido sustituido en la nueva versión del CSV por el siguiente: «Sufrir agresiones físicas de su pareja». ${ }^{\mathrm{b}}$ Este ítem ha sido sustituido en la actual versión del CSV por el siguiente: «Ser denunciado/a por malos tratos por su pareja». ${ }^{\mathrm{c}}$ Este ítem ha sido eliminado en la nueva versión del CSV. Se indican las diferencias entre las mujeres y los varones según la prueba de $\chi^{2}$ de tablas de contingencia. $* p<.05$. $* * p<.01 . * * * p<.001$. 
Tabla 3. Impacto del estrés (nivel de distrés) asociado a cada uno de los sucesos vitales ( $M$ y $D T$ ) (Muestras 1 y 2, $N=1.851$ )

\begin{tabular}{|c|c|c|c|}
\hline Número de ítem del CSV & $\begin{array}{c}\text { Total } \\
(N=1.851)\end{array}$ & $\begin{array}{c}\text { Mujeres } \\
(N=1.392)\end{array}$ & $\begin{array}{l}\text { Hombres } \\
(N=459)\end{array}$ \\
\hline 21. Muerte de un hijo & $3.33(1.21)$ & $3.20(1.30)$ & $4.00(0.00)$ \\
\hline 13. Muerte del cónyuge & $3.08(0.94)$ & $2.75(1.03)$ & $3.60(0.54)$ \\
\hline 31. Muerte de algún familiar cercano (padre, hermano, etc.) & $2.28(1.07)$ & $3.00(1.04)^{* * *}$ & $2.43(1.07)$ \\
\hline 20. Muerte de un amigo íntimo & $2.77(0.90)$ & $2.85(0.92)$ & $2.55(0.79)$ \\
\hline 08. Divorcio o separación & $2.72(1.08)$ & $2.74(1.07)$ & $2.65(1.16)$ \\
\hline 19. Ruptura de noviazgo o relación similar & $2.61(1.02)$ & $2.67(1.00)^{* *}$ & $2.36(1.05)$ \\
\hline 59. Discusión especialmente grave con su cónyuge o pareja & $2.56(1.01)$ & $2.65(1.01) * * *$ & $2.22(0.95)$ \\
\hline 28. Abortar & $2.56(1.12)$ & $2.56(1.12)$ & - \\
\hline 09. Problema legal grave (con o sin cárcel) & $2.48(1.09)$ & $2.53(1.10)$ & $2.40(1.10)$ \\
\hline 45. Enfermedad de algún familiar & $2.47(0.97)$ & $2.54(0.98) * * *$ & $2.23(0.93)$ \\
\hline 16. Sufrir malos tratos de su pareja & $2.40(1.14)$ & $2.64(1.12) * * *$ & $1.71(0.90)$ \\
\hline 01.Quedarse sin trabajo & $2.39(1.02)$ & $2.46(1.04)^{* *}$ & $2.10(0.91)$ \\
\hline 10. Exámenes importantes & $2.34(0.91)$ & $2.43(0.90) * * *$ & $2.06(0.86)$ \\
\hline 50. Separación de los padres & $2.34(1.00)$ & $2.34(1.01)$ & $2.35(0.98)$ \\
\hline 52. Hospitalización de algún familiar & $2.33(1.00)$ & $2.44(0.99) * * *$ & $2.00(0.96)$ \\
\hline 51. Fracaso en algún negocio importante & $2.30(1.03)$ & $2.45(1.03)^{*}$ & $2.02(1.00)$ \\
\hline 53. Sufrir asalto o situación de violencia física & $2.28(1.08)$ & $2.42(1.04)$ & $1.96(1.10)$ \\
\hline 24. Complicación legal en una pelea & $2.26(0.92)$ & $2.28(0.88)$ & $2.12(1.05)$ \\
\hline 11. Reducción sustancial en los ingresos & $2.23(0.96)$ & $2.28(0.96)^{* *}$ & $2.07(0.93)$ \\
\hline 29. Aborto de su mujer o pareja & $2.38(1.06)$ & - & $2.38(1.06)$ \\
\hline 02. Enfermedad prolongada que requiere tratamiento & $2.20(1.00)$ & $2.23(1.02)$ & $2.10(0.96)$ \\
\hline 54. Pérdidas económicas no asociadas al trabajo & $2.17(0.95)$ & $2.22(0.95)$ & $2.06(0.92)$ \\
\hline 41. Suspender exámenes & $2.17(0.99)$ & $2.23(1.00) * * *$ & $1.95(0.92)$ \\
\hline 06. Problemas con los hijos (no poder verlos por separación, algún problema grave en el colegio, etc.) & $2.13(1.06)$ & $2.17(1.08)$ & $2.04(0.99)$ \\
\hline 40. Lesión física importante & $2.12(0.92)$ & $2.12(0.92)$ & $2.11(0.96)$ \\
\hline 34. Sufrir robo & $2.11(0.98)$ & $2.20(1.01)^{*}$ & $1.83(0.85)$ \\
\hline 26. Cambio de casa o tener que buscar una nueva vivienda & $2.06(0.96)$ & $2.23(0.97)^{*}$ & $1.86(0.88)$ \\
\hline 23. Hijos lejos del hogar & $2.06(0.94)$ & $2.01(0.96)$ & $2.16(0.95)$ \\
\hline 17 Comprar una casa & $2.02(0.91)$ & $2.01(0.90)$ & $2.03(0.95)$ \\
\hline 56. Problemas con superiores en el trabajo & $2.00(0.95)$ & $2.07(0.99)^{* *}$ & $1.83(0.83)$ \\
\hline 44. Embarazo de su mujer o pareja & $2.00(1.00)$ & - & $2.08(1.01)$ \\
\hline 33. Préstamo o hipoteca de más de $100.000 €$ & $1.99(0.99)$ & $2.02(1.00)$ & $1.91(0.97)$ \\
\hline 04. Retiro laboral (jubilarse) & $1.98(0.96)$ & $2.09(0.98) * * *$ & $1.47(0.67)$ \\
\hline 03. Problemas con colegas o compañeros de trabajo & $1.97(0.94)$ & $2.04(0.97) * * *$ & $1.75(0.81)$ \\
\hline 38. Accidente de tráfico & $1.96(0.91)$ & $2.04(0.93)^{*}$ & $1.71(0.82)$ \\
\hline 60. Alquilar una vivienda ${ }^{\text {a }}$ & $1.90(1.10)$ & $2.14(1.21)$ & $1.33(0.57)$ \\
\hline 42. Quedarse embarazada & $1.90(1.00)$ & $1.90(1.00)$ & - \\
\hline 27. Problemas con vecinos o con familiares que no viven en la casa familiar & $1.88(0.91)$ & $1.93(0.93)^{* *}$ & $1.72(0.84)$ \\
\hline 49. Reconciliación matrimonial o de pareja & $1.80(0.92)$ & $1.85(0.92)$ & $1.60(0.89)$ \\
\hline 47. Cambio de horario o de las condiciones en el trabajo actual & $1.75(0.87)$ & $1.83(0.91) * * *$ & $1.52(0.66)$ \\
\hline 36. Enfermedad o accidente que requiere guardar cama & $1.74(0.92)$ & $1.80(0.96)^{*}$ & $1.59(0.79)$ \\
\hline 46. Ruptura de relaciones sexuales fuera matrimonio & $1.72(0.92)$ & $1.73(0.89)$ & $1.71(1.01)$ \\
\hline 39. Repentino y serio deterioro de la audición o visión & $1.72(0.96)$ & $1.70(0.96)$ & $1.78(0.94)$ \\
\hline 18. Cambio de lugar de trabajo & $1.71(0.87)$ & $1.73(0.90)$ & $1.69(0.80)$ \\
\hline 43. Enamorarse o iniciar amistad íntima y profunda & $1.71(0.90)$ & $1.71(0.90)$ & $1.74(0.89)$ \\
\hline 30. Nuevo empleo laboral & $1.70(0.83)$ & $1.72(0.83)$ & $1.63(0.82)$ \\
\hline 37. Finalización de estudios & $1.69(0.92)$ & $1.73(0.92)$ & $1.56(0.90)$ \\
\hline 48. Vivir temporalmente fuera del hogar & $1.68(0.91)$ & $1.71(0.95)$ & $1.61(0.78)$ \\
\hline 14. Problemas legales relacionados con el alcohol o las drogas & $1.66(0.86)$ & $1.65(0.90)$ & $1.67(0.82)$ \\
\hline 58. El cónyuge comienza a trabajar fuera de casa & $1.63(0.93)$ & $1.75(0.99)$ & $1.19(0.39)$ \\
\hline 15. Ascenso en el trabajo & $1.63(0.84)$ & $1.63(0.82)$ & $1.63(0.88)$ \\
\hline 12. Reformas en la casa & $1.61(0.81)$ & $1.62(0.81)$ & $1.57(0.81)$ \\
\hline 07. Nacimiento de un hijo & $1.57(0.90)$ & $1.63(0.99)$ & $1.46(0.76)$ \\
\hline 22. Relaciones sexuales fuera del matrimonio & $1.52(0.86)$ & $1.52(0.82)$ & $1.53(0.96)$ \\
\hline 05. Matrimonio & $1.52(0.82)$ & $1.54(0.83)$ & $1.47(0.79)$ \\
\hline 55. Cambio de centro de estudios & $1.47(0.79)$ & $1.52(0.80)^{*}$ & $1.29(0.73)$ \\
\hline 57. Éxito personal importante & $1.47(0.77)$ & $1.48(0.78)$ & $1.42(0.75)$ \\
\hline 25. Problema legal menor (multa, etc.) & $1.45(0.71)$ & $1.48(0.74)^{*}$ & $1.26(0.61)$ \\
\hline 35. Cambio en las costumbres personales (salir, vestir, etc.) ${ }^{b}$ & $1.40(0.66)$ & $1.41(0.69)$ & $1.35(0.55)$ \\
\hline 32. Período de fiestas ${ }^{c}$ & $1.28(0.60)$ & $1.30(0.62)^{* *}$ & $1.21(0.52)$ \\
\hline
\end{tabular}

Nota . Ítems 21-28 = elevado nivel de estrés, 9-44 moderado-alto nivel de estrés, resto = bajo nivel de estrés. ${ }^{a}$ Este ítem ha sido eliminado en la nueva versión del CSV. ${ }^{\mathrm{b}}$ Este ítem ha sido sustituido en la nueva versión del CSV por el siguiente: «Ser denunciado/a por malos tratos por su pareja». ${ }^{\mathrm{c}}$ Este ítem ha sido sustituido en la actual versión del CSV por el siguiente: «Sufrir agresiones físicas de su pareja». Se indican las diferencias entre las mujeres y los varones según la prueba de $t$ de Student. ${ }^{*} p<.05 . * * p<.01 . * * * p<.001$. 
Tabla 4. Análisis factorial exploratorio del CSV. Estructura factorial basada en los 42 ítems seleccionados. Se indican también las correlaciones corregidas ítem-escala $(r)$ (Muestra 1, $N=867$ )

\begin{tabular}{|c|c|c|}
\hline Item $C S V^{a}$ & Peso factorial & $r$ \\
\hline \multicolumn{3}{|l|}{ Factor 1. Trabajo } \\
\hline 03. Problemas con colegas o compañeros de trabajo & .52 & .31 \\
\hline 15. Ascenso en el trabajo & .38 & .30 \\
\hline 18. Cambio de lugar de trabajo & .60 & .45 \\
\hline 30. Nuevo empleo laboral ${ }^{4}$ & .54 & .40 \\
\hline 47. Cambio de horario o de las condiciones en el trabajo actual & .68 & .49 \\
\hline 56. Problemas con superiores en el trabajo & .70 & .38 \\
\hline 57. Éxito personal importante ${ }^{2}$ & .30 & .25 \\
\hline \multicolumn{3}{|l|}{ Factor 2. Relaciones de pareja } \\
\hline 08. Divorcio o separación ${ }^{7}$ & .42 & .32 \\
\hline 16. Sufrir malos tratos por su pareja & .48 & .40 \\
\hline 19. Ruptura de noviazgo o relación similar & 69 & .53 \\
\hline 22. Relaciones sexuales fuera del matrimonio & .42 & .30 \\
\hline 43. Enamorarse o iniciar una amistad íntima y profunda & .50 & .37 \\
\hline 46. Ruptura de relaciones sexuales fuera del matrimonio & .69 & .31 \\
\hline 49. Reconciliación matrimonial o de pareja & .58 & .36 \\
\hline 59. Discusión especialmente grave con su cónyuge o pareja & .60 & .42 \\
\hline \multicolumn{3}{|l|}{ Factor 3. Vivienda/Legal/Social } \\
\hline 12. Reformas en la casa ${ }^{7}$ & .45 & .32 \\
\hline 14. Problemas legales relacionados con alcohol o drogas ${ }^{2,6}$ & .30 & .21 \\
\hline 17. Comprar una casa & .68 & .31 \\
\hline 25. Problema legal menor (multa, etc.) & .30 & .23 \\
\hline 26. Cambio de casa o tener que comprar nueva vivienda & .59 & .35 \\
\hline 27. Problemas con vecinos o con familiares que no viven en la casa familiar & .32 & .24 \\
\hline 34. Sufrir robo & .39 & .32 \\
\hline 48. Vivir temporalmente fuera del hogar & .47 & .37 \\
\hline 50. Separación de los padres & .31 & .24 \\
\hline 53. Sufrir asalto o situación de violencia física & .34 & .28 \\
\hline \multicolumn{3}{|l|}{ Factor 4. Pérdida laboral/ingresos } \\
\hline 01. Quedase sin trabajo & .75 & .53 \\
\hline 04. Retiro laboral (jubilación) & .61 & .36 \\
\hline 11. Reducción sustancial en los ingresos & .69 & .60 \\
\hline 51. Fracaso en algún negocio importante & .37 & .28 \\
\hline 54. Pérdidas económicas importantes no asociadas al trabajo & .33 & .32 \\
\hline \multicolumn{3}{|l|}{ Factor 5. Salud familia } \\
\hline 31. Muerte de algún familiar cercano (padre, hermano, etc.) & .60 & .41 \\
\hline 45. Enfermedad de algún familiar & .85 & .64 \\
\hline 52. Hospitalización de algún familiar & .84 & .62 \\
\hline \multicolumn{3}{|l|}{ Factor 6. Salud propia } \\
\hline 2. Enfermedad prolongada que requiere tratamiento & .66 & .35 \\
\hline 36. Enfermedad o accidente que requiere guardar cama & .72 & .46 \\
\hline 38. Accidente de tráfico & .31 & .30 \\
\hline 39. Repentino y serio deterioro de la audición o visión & .30 & .27 \\
\hline 40. Lesión física importante & .72 & .48 \\
\hline \multicolumn{3}{|l|}{ Factor 7. Familia/Hijos } \\
\hline 05. Matrimonio & .36 & .29 \\
\hline 06. Problemas con los hijos (por separación, etc.) & .69 & .40 \\
\hline 23. Hijos lejos del hogar & .43 & .21 \\
\hline 33. Préstamo o hipoteca de más de $100.000 €$ & .48 & .23 \\
\hline
\end{tabular}

Nota. Los superíndices que aparecen en algunos ítems indican que, en el análisis factorial exploratorio, el ítem saturaba también en el factor que corresponda al número indicado en el superíndice. ${ }^{a}$ Número de ítem como aparece en el CSV original de 60 ítems. Ítems del CSV no incluidos en el análisis final: 7, 9, 10, 13, 20, 21, 24, 28, 29, 32, 35, 37, 41, 42, 44, 55, 58, 60. 
propia», 5 ítems) hace alusión a problemas importantes relacionados con la salud propia. Finalmente, el séptimo factor («Familia/hijos», 4 ítems) es un factor sobre eventos asociados a la familia y los hijos.

En general, las correlaciones entre los factores fueron bajas y moderadas-bajas (el rango de correlaciones fue entre .03 y .35). Las correlaciones más altas se die- ron entre el factor de trabajo y los factores de pérdida laboral/ingresos y vivienda/legal/social. Este último factor también correlacionó de forma moderada con el factor de pérdida laboral/ingresos. Las correlaciones más bajas fueron con el factor 7 (el rango de correlaciones de este factor con el resto varió entre .03 y .18) (véase la Tabla 5).

Tabla 5. Correlaciones entre los factores del CSV-42 (análisis de componentes principales; mitad inferior izquierda), coeficiente alfa de Cronbach ${ }^{\mathrm{a}}$ (en negrita, en la diagonal), y correlaciones entre las escalas ${ }^{\mathrm{b}}$ (mitad superior derecha)

\begin{tabular}{lccccccc}
\hline & Factor 1 & Factor 2 & Factor 3 & Factor 4 & Factor 5 & Factor 6 & Factor 7 \\
\cline { 2 - 7 } Factor 1: Trabajo &. $\mathbf{7 6}$ & .27 & .36 & .29 & .13 & .12 & .08 \\
Factor 2: Relaciones de pareja & .20 & $\mathbf{. 8 4}$ & .33 & .20 & .12 & .20 & .11 \\
Factor 3: Vivienda/Legal/Social & .35 & .25 &. $\mathbf{7 8}$ & .28 & .18 & .21 & .10 \\
Factor 4: Pérdida laboral/ingresos & .33 & .16 & .29 & .75 & .11 & .19 & .23 \\
Factor 5: Salud familia & .10 & .13 & .16 & .07 & .71 & .20 & .05 \\
Factor 6: Salud propia & .08 & .16 & .12 & .10 & .17 & .71 & .13 \\
Factor 7: Familia/Hijos & .14 & .04 & .15 & .18 & .03 & .06 & .70 \\
\hline
\end{tabular}

Nota. ${ }^{\text {a }}$ Los coeficientes alfa se basan en correlaciones policóricas. ${ }^{\mathrm{b}}$ Las correlaciones entre las escalas se basan en la muestra total $(N=1.851)$; el resto se basa en la Muestra $1(N=867)$. Son significativas $(p<.01)$ las correlaciones a partir del valor $\geq .10$.

\section{Análisis factorial confirmatorio}

Con objeto de probar empíricamente y de forma transversal la validez de la estructura factorial de 7 factores del CSV obtenida mediante análisis factorial exploratorio, llevamos a cabo un análisis factorial confirmatorio basándonos en una muestra independiente de un mismo tipo de población (Muestra 2). El modelo examinado se especificó partiendo de los 42 indicadores y los 7 factores latentes obtenidos a partir del análisis exploratorio (véase la Tabla 4) (se definieron como factores correlacionados). Tras aplicar el test Lagranje multiplier los índices de modificación indicaron la conveniencia de liberar algunas de las constricciones impuestas al modelo, i.e., especificando algunas covariaciones entre residuos como libres (que inicialmente fueron especificadas como fijas). En este sentido, se liberaron los siguientes pares de covariaciones de residuos: E56-E3, E33-E17, E49-E59, E54-E1, y E57-E15. Esta modificación da un sentido menos artificial al modelo, ya que las parejas de residuos indicados comparten conceptualmente cierto nivel de variabilidad común asociada a relaciones específicas entre las variables correspondientes.

Tras someter a prueba este modelo final constatamos que en general se daba un buen ajuste a los datos, obteniéndose los siguientes valores en los índices de bondad de ajuste examinados: $S-B \chi^{2}=1176.3$ (710), $S-B \chi^{2} / g 1=$ $1.65, \mathrm{CFI}=0.96, \mathrm{SRMR}=0.099, \mathrm{y}$ RMSEA $=0.026(\mathrm{IC}$
$95 \%=0.023-0.028)$. Con la excepción del SRMR, todos los demás indicadores sobre el ajuste del modelo a los datos indican un ajuste entre bueno y excelente. Las estimaciones paramétricas de las ecuaciones de medida fueron todas estadísticamente significativas, variando entre .31 y .91 (el 76\% de los ítems tenían valores superiores a .50). En consecuencia, entendimos que los 7 factores de este conjunto de 42 ítems del CSV representaban adecuadamente las 7 dimensiones de estrés psicosocial aisladas previamente.

\section{Fiabilidad y correlaciones entre las escalas}

Se evaluó la consistencia interna (coeficiente alfa de Cronbach) de las escalas de estrés psicosocial (Tabla 5), así como también las correlaciones corregidas entre cada ítem y la puntuación total en la escala correspondiente (Tabla 4). Los coeficientes alfa, basados en correlaciones policóricas, fueron todos $\geq .70$ (rango .70-.84). Las correlaciones corregidas ítem-escala variaron entre $.21 \mathrm{y}$ .64 (suele asumirse como valor aceptable a partir de .20). Los coeficientes alfa de la escala total fueron .82 (CSV) y .79 (CSV-44).

El patrón de correlaciones entre las escalas fue bastante similar al de las correlaciones entre los factores, si bien las correlaciones entre las primeras fueron ligeramente superiores (el rango de correlaciones varió entre .05 y .36). Las correlaciones más bajas también correspondieron al factor (escala) 7, variando entre .05 y .23 . 
Estadísticos descriptivos y diferencias entre mujeres y varones

Las medias y desviaciones típicas correspondientes a las diferentes escalas se indican de forma separada en función de los grupos de mujeres y varones y de la muestra total para cada una de las variables (véase la Tabla 6). Tras aplicar análisis de varianza univariados constatamos diferencias significativas entre mujeres y varones para tres de las escalas de estrés psicosocial (i.e., relaciones de pareja, pérdida laboral/ingresos, y salud de la familia). Las mujeres puntuaron significativamente más alto que los hombres en estas tres variables. No obstante, la magnitud del tamaño del efecto ( $\eta^{2}$ parcial) fue bajo (no superó el 2\% de la varianza). También encontramos diferencias significativas entre mujeres y varones en la puntuación total en estrés percibido (tanto en la puntuación del CSV como en la del CSV-42). En ambos casos, las mujeres informaron haber experimentado mayor grado de estrés que los hombres.

Con objeto de proporcionar información normativa sobre el CSV, presentamos en la Tabla 7 los puntos de corte (percentiles) correspondientes al CSV completo y al CSV-42, separando los valores correspondientes a la muestra total y a los grupos de varones y mujeres.

\section{Validez convergente y discriminante}

Calculamos la validez convergente y discriminante del CSV y CSV-42 correlacionando las puntuaciones totales de ambas formas (estrés percibido y frecuencia de sucesos vitales), así como también las puntuaciones en las escalas del CSV-42, con varios constructos psicológicos que se suponen relacionados (síntomas psicopatológicos, ansiedad, depresión, y afecto negativo) o no relacionados (afecto positivo) con el estrés psicosocial. Los resultados de estas correlaciones se indican en la Tabla 8.

La inspección de la tabla refleja correlaciones significativas entre las variables de estrés y las variables psicopatológicas y de la afectividad. Como puede apreciarse en dicha tabla, un primer patrón consiste en correlaciones positivas y significativas entre estrés percibido y síntomas psicopatológicos (incluidas la ansiedad y la depresión) y afectividad negativa; aunque la frecuencia de SV también correlaciona con estas variables psicológicas, las correlaciones tienden a ser más bajas. Así mismo, se observa que el estrés no correlaciona con el afecto positivo (las correlaciones son muy bajas o inexistentes). En lo que concierne a las correlaciones entre las dimensiones de estrés psicosocial y las distintas variables psicológicas se constatan correlaciones moderadas-ba- jas, excepto con el afecto positivo cuyas correlaciones son irrelevantes. También encontramos un patrón de muy bajas correlaciones con las dimensiones de salud/ familia y familia/hijos. Este patrón se repite con todas las variables psicopatológicas y con la afectividad negativa.

\section{Validez predictiva}

Para examinar la contribución del CSV en la predicción de las variables psicopatológicas aplicamos un diseño general de análisis de regresión múltiple en el que las variables independientes se incluyeron simultáneamente en la ecuación y consistieron en el estrés percibido junto con las variables sociodemográficas (edad y género) y de afectividad (afecto positivo y negativo). Este diseño se aplicó en dos series de análisis de regresión, según que se utilizase el CSV o el CSV-42 como variable independiente. Cada serie (4 análisis de regresión) se aplicó de forma separada para las cuatro variables dependientes estudiadas (i.e., DSM-5 transversal, ansiedad, depresión, y enfermedades). Este tipo de análisis permite examinar la especificidad predictiva del estrés psicosocial, controlando el efecto de las variables sociodemográficas y de personalidad (afectividad).

En la Tabla 9 se exponen los resultados derivados de la primera serie de análisis de regresión. Como puede apreciarse en la tabla, el estrés percibido contribuye de forma significativa en la predicción de todas y cada una de las variables dependientes estudiadas, aunque, partiendo de los valores de beta, el poder predictivo del estrés es claramente superior para las variables de salud mental (i.e., síntomas DSM-5 generales, ansiedad y depresión) que para la enfermedad. Puesto que esta predicción es estadísticamente significativa, el CSV proporciona un nivel relevante de validez incremental en la predicción de los síntomas psicopatológicos y las enfermedades (véase la Tabla 9). Las restantes variables independientes parecen jugar un papel menos consistente, excepto la afectividad negativa cuyo efecto es significativo en todos los análisis y parece ser la variable más relevante considerando sus valores de beta. También se constata que la varianza total explicada por el modelo de regresión es notablemente menor cuando se trata de predecir las enfermedades que cuando se refiere a la sintomatología psicológica (34-38\% vs. 8\%).

Los resultados relacionados con la segunda serie de análisis de regresión están indicados en la Tabla 10 (ahora la variable independiente de estrés psicosocial es el CSV-42). De estos resultados se desprende un patrón similar al referido a los resultados presentados en la Tabla 9 , si bien la varianza explicada es ligeramente infe- 
Tabla 6. Medias y desviaciones típicas (entre paréntesis) en las variables de estrés psicosocial y en las variables psicopatológicas y de afectividad en función del género (muestra total)

\begin{tabular}{|c|c|c|c|c|c|}
\hline & $\begin{array}{c}\text { Total } \\
(N=1.851)\end{array}$ & $\begin{array}{c}\text { Mujeres } \\
(n=1.392)\end{array}$ & $\begin{array}{c}\text { Varones } \\
(n=459)\end{array}$ & $\begin{array}{c}\text { Mujeres vs. } \\
\text { Varones }\end{array}$ & \\
\hline Variable $^{\mathrm{a}}$ & Media $(D T)$ & Media $(D T)$ & Media $(D T)$ & $F(1.1849)$ & $\eta^{2}$ parcial \\
\hline \multicolumn{6}{|l|}{ CSV-42: } \\
\hline Trabajo (0-28) & $3.84(3.51)$ & $3.88(2.82)$ & $3.68(2.82)$ & 1.1 & .01 \\
\hline Relaciones de pareja (0-32) & $3.20(3.76)$ & $3.40(3.36)$ & $2.63(3.37)$ & $13.5 * * *$ & .01 \\
\hline Vivienda/Legal/Social (0-40) & $3.42(3.53)$ & $3.43(3.58)$ & $3.38(3.38)$ & 0.1 & .00 \\
\hline Pérdida laboral/ingresos $(0-20)$ & $2.60(3.10)$ & $2.73(3.20)$ & $2.23(2.71)$ & $9.1 * *$ & .01 \\
\hline Salud familia $(0-12)$ & $2.80(3.14)$ & $2.95(3.25)$ & $2.35(2.75)$ & $12.9 * * *$ & .01 \\
\hline Salud propia $(0-20)$ & $1.48(2.40)$ & $1.49(2.41)$ & $1.46(2.34)$ & 0.1 & .00 \\
\hline Familia/Hijos (0-16) & $.95(1.75)$ & $.93(1.74)$ & $.97(1.55)$ & 0.3 & .00 \\
\hline Estrés percibido total $(0-168)$ & $18.25(12.10)$ & $18.75(12.30)$ & $16.70(11.24)$ & $10.3 * *$ & .02 \\
\hline Frecuencia SV total (0-42) & $9.05(5.00)$ & $9.02(4.96)$ & $9.10(5.05)$ & 0.6 & .00 \\
\hline \multicolumn{6}{|l|}{ CSV: } \\
\hline Estrés percibido total (0-228) & $23.30(14.00)$ & $23.97(14.15)$ & $21.20(13.23)$ & $14.5 * * *$ & .02 \\
\hline Frecuencia SV total $(0-57)$ & $11.38(5.68)$ & $11.36(5.65)$ & $11.40(5.70)$ & 0.1 & .00 \\
\hline DSM-5 Transversal (0-92) & $13.96(9.20)$ & $13.92(8.82)$ & $14.12(9.97)$ & 5.14 & .00 \\
\hline Escala de Ansiedad (EA) (0-40) & $10.80(8.10)$ & $11.26(8.10)$ & $9.31(8.10)$ & $7.24 * *$ & .02 \\
\hline Cuestionario de Depresión (CD) (0-64) & $7.40(8.40)$ & $7.50(8.50)$ & $7.10(8.10)$ & 0.28 & .00 \\
\hline Afecto positivo (PANAS-AP) $(0-50)$ & $31.10(7.10)$ & $31.12(7.00)$ & $30.85(6.85)$ & 0.30 & .00 \\
\hline Afecto negativo (PANAS-AN) (0-50) & $19.05(6.20)$ & $19.26(6.20)$ & $18.30(6.08)$ & $4.40^{*}$ & .01 \\
\hline
\end{tabular}

Nota. ${ }^{a}$ Entre paréntesis se indica el rango posible de valores de cada variable. No se incluyen los Ítems 32,35 y 60 tras haber sido modificados o suprimido (Ítem 60) de la versión actual. * $p<0.05 ; * * p<0.01 ; * * * p<0.001$.

Tabla 7. Percentiles (puntos de corte) para las puntuaciones en el CSV y CSV-42 (muestra total)

\begin{tabular}{|c|c|c|c|c|c|c|}
\hline \multirow[b]{2}{*}{ Percentil } & \multicolumn{3}{|c|}{$\mathrm{CSV}^{\mathrm{a}}$} & \multicolumn{3}{|c|}{ CSV-42 } \\
\hline & Total & Mujeres & Varones & Total & Mujeres & Varones \\
\hline 5 & 7 & 7 & 6 & 4 & 4 & 3 \\
\hline 10 & 8 & 9 & 8 & 5 & 6 & 5 \\
\hline 15 & 11 & 11 & 9 & 7 & 7 & 6 \\
\hline 20 & 12 & 13 & 11 & 8 & 9 & 8 \\
\hline 25 & 14 & 14 & 12 & 10 & 10 & 9 \\
\hline 30 & 15 & 16 & 13 & 11 & 11 & 10 \\
\hline 35 & 16 & 17 & 14 & 12 & 13 & 11 \\
\hline 40 & 18 & 18 & 16 & 13 & 14 & 12 \\
\hline 45 & 19 & 20 & 17 & 15 & 15 & 13 \\
\hline 50 & 20 & 21 & 19 & 16 & 17 & 14 \\
\hline 55 & 22 & 23 & 20 & 17 & 18 & 15 \\
\hline 60 & 23 & 24 & 21 & 19 & 19 & 17 \\
\hline 65 & 25 & 26 & 23 & 20 & 21 & 18 \\
\hline 70 & 27 & 28 & 24 & 22 & 22 & 20 \\
\hline 75 & 30 & 31 & 27 & 24 & 24 & 22 \\
\hline 80 & 32 & 33 & 30 & 26 & 27 & 24 \\
\hline 85 & 36 & 37 & 33 & 30 & 31 & 26 \\
\hline 90 & 42 & 42 & 39 & 34 & 35 & 31 \\
\hline 95 & 50 & 52 & 47 & 42 & 42 & 39 \\
\hline
\end{tabular}

Nota. $N$ total $=1.851$, mujeres $n=1.392$, varones $n=459$. ${ }^{\text {a }}$ No se incluyen los Ítems 32,35 y 60 tras haber sido modificados o suprimido (Ítem 60) de la versión actual. 
Tabla 8. Correlaciones entre las variables de estrés psicosocial y las variables psicopatológicas y de afectividad (Submuestra 2, $n=739)^{\text {a }}$

\begin{tabular}{|c|c|c|c|c|c|c|}
\hline Variable de estrés psicosocial & $\begin{array}{c}\text { Síntomas } \\
\text { DSM-5 }\end{array}$ & EA & $\mathrm{CD}$ & $\begin{array}{c}\text { PANAS } \\
\text { AP }\end{array}$ & $\begin{array}{c}\text { PANAS } \\
\text { AN }\end{array}$ & Enfermedades \\
\hline \multicolumn{7}{|l|}{ CSV-42 (estrés percibido) } \\
\hline Trabajo & $.16^{* * *}$ & $.17 * * *$ & $.12 * *$ & -.01 & $.17 * * *$ & $.08^{*}$ \\
\hline Relaciones de pareja & $.23 * * *$ & $.22 * * *$ & $.21 * * *$ & -.02 & $.20 * * *$ & $.10 * *$ \\
\hline Vivienda/Legal/Social & $.25 * * *$ & $.20 * * *$ & $.24 * * *$ & -.05 & $.20 * * *$ & $.07 *$ \\
\hline Pérdida laboral/ingresos & $.17 * * *$ & $.18 * * *$ & $.23 * * *$ & -.02 & $.15^{* * *}$ & $.08^{*}$ \\
\hline Salud familia & $.08 *$ & $.10 * *$ & .05 & -.01 & .06 & $.09 *$ \\
\hline Salud propia & $.17 * * *$ & $.11 * *$ & $.14 * * *$ & .00 & $.16^{* * *}$ & - \\
\hline Familia/Hijos & .07 & .01 & .02 & .04 & .01 & .03 \\
\hline CSV-42 Total & $.28 * * *$ & $.27 * * *$ & $.26^{* * *}$ & -.01 & $.25 * * *$ & $.16 * * * b$ \\
\hline CSV-42 (frecuencia de SV) & $.20 * * *$ & $.16^{* * *}$ & $.14 * * *$ & .04 & $.14 * * *$ & $.13 * * * b$ \\
\hline CSV (estrés percibido) & $.32 * * *$ & $.31 * * *$ & $.30 * * *$ & -.01 & $.27 * * *$ & $.16 * * * b$ \\
\hline CSV (frecuencia de SV) & $.21 * * *$ & $.17 * * *$ & $.15 * * *$ & .06 & $.13 * * *$ & $.13 * * * b$ \\
\hline
\end{tabular}

Nota. ${ }^{\text {a }}$ Parte de la Muestra 2 que cumplimentó el protocolo también en una segunda ocasión 6 meses más tarde. ${ }^{\mathrm{b}}$ Se eliminaron los ítems del CSV referidos a salud propia (enfermedades). CD = Cuestionario de Depresión; EA = Escala de Ansiedad; PANAS-AN = afecto negativo; PANAS-AP $=$ afecto positivo; $* p<0.05 ; * * p<0.01 ; * * * p<0.001$.

Tabla 9. Análisis de regresión múltiple: Contribución del estrés psicosocial percibido (CSV), referido al último año, en la predicción de los síntomas psicopatológicos, la ansiedad, la depresión y las enfermedades 6 meses después, controlando el efecto de la edad, el género y la afectividad positiva y negativa (Submuestra $2, n=739)^{\text {a }}$

\begin{tabular}{|c|c|c|c|c|}
\hline Variable & $R^{2}$ & $B$ & $E T B$ & Beta \\
\hline DSM-5 Transversal & $.34 * * *$ & & & \\
\hline Edad & & -.13 & .03 & $-.14 * * *$ \\
\hline Género & & 1.53 & .68 & $.07 *$ \\
\hline PANAS-AP & & -.22 & .05 & $-.17 * * *$ \\
\hline PANAS-AN & & .58 & .04 & $.39 * * *$ \\
\hline CSV (estrés percibido) & & .14 & .02 & $.19 * * *$ \\
\hline Ansiedad (EA) & $.38 * * *$ & & & \\
\hline Edad & & -.06 & .03 & $-.07 *$ \\
\hline Género & & .95 & .58 & .05 \\
\hline PANAS-AP & & -.04 & .04 & -.04 \\
\hline PANAS-AN & & .69 & .05 & $.53 * * *$ \\
\hline CSV (estrés percibido) & & .18 & .02 & $.14^{* * *}$ \\
\hline Depresión (CD) & $.34 * * *$ & & & \\
\hline Edad & & -.04 & .03 & -.05 \\
\hline Género & & .50 & .62 & .04 \\
\hline PANAS-AP & & -.26 & .04 & $-.21 * * *$ \\
\hline PANAS-AN & & .52 & .05 & $.38 * * *$ \\
\hline CSV (estrés percibido) & & .12 & .02 & $.20 * * *$ \\
\hline Enfermedades & $.08 * * *$ & & & \\
\hline Edad & & -.02 & .00 & -.06 \\
\hline Género & & .60 & .16 & $.12 * * *$ \\
\hline PANAS-AP & & -.03 & .01 & $-.07 *$ \\
\hline PANAS-AN & & .04 & .01 & $.13 * * *$ \\
\hline CSV (estrés percibido) ${ }^{\mathrm{b}}$ & & .03 & .00 & $.11 * *$ \\
\hline
\end{tabular}

Nota: ${ }^{a}$ Consiste en la parte de la Muestra 2 que también cumplimentó el protocolo en una segunda ocasión 6 meses más tarde. ${ }^{\mathrm{b}}$ Se eliminaron los ítems del CSV referidos a salud propia (enfermedades). CD = Cuestionario de Depresión; EA = Escala de Ansiedad; PANAS-AN $=$ afecto negativo; PANAS-AP $=$ afecto positivo. ${ }^{*} p<0.05 ; * * p<0.01 ; * * * p<0.001$. 
Tabla 10. Análisis de regresión múltiple: Contribución del estrés psicosocial percibido (CSV-42), referido al último año, en la predicción de los síntomas psicopatológicos, la ansiedad, la depresión y las enfermedades 6 meses después, controlando el efecto de la edad, el género y la afectividad positiva y negativa (Submuestra $2, n=739)^{\text {a }}$

\begin{tabular}{|c|c|c|c|c|}
\hline Variable & $R^{2}$ & $B$ & $E T B$ & Beta \\
\hline DSM-5 Transversal & $.33 * * *$ & & & \\
\hline Edad & & -.14 & .03 & $-.15 * * *$ \\
\hline Género & & 1.10 & .68 & $.07 *$ \\
\hline PANAS-AP & & -.21 & .04 & $-.17 * * *$ \\
\hline PANAS-AN & & .66 & .05 & $.40 * * *$ \\
\hline CSV-42 (estrés percibido) & & .15 & .03 & $.17 * * *$ \\
\hline Ansiedad (EA) & $.37 * * *$ & & & \\
\hline Edad & & -.07 & .02 & $-.08 * *$ \\
\hline Género & & .99 & .58 & .05 \\
\hline PANAS-AP & & -.04 & .04 & -.04 \\
\hline PANAS-AN & & .70 & .04 & $.54 * * *$ \\
\hline CSV-42 (estrés percibido) & & .10 & .02 & $.12 * * *$ \\
\hline Depresión (CD) & $.32 * * *$ & & & \\
\hline Edad & & -.05 & .03 & -.06 \\
\hline Género & & .43 & .63 & .02 \\
\hline PANAS-AP & & -.26 & .04 & $-.21 * * *$ \\
\hline PANAS-AN & & .54 & .05 & $.39 * * *$ \\
\hline CSV-42 (estrés percibido) & & .13 & .02 & $.16^{* * *}$ \\
\hline Enfermedades & $.07 * * *$ & & & \\
\hline Edad & & -.01 & .01 & -.05 \\
\hline Género & & .60 & .16 & $.12 * * *$ \\
\hline PANAS-AP & & -.02 & .01 & -.06 \\
\hline PANAS-AN & & .05 & .01 & $.13^{* * *}$ \\
\hline CSV-42 (estrés percibido) ${ }^{\mathrm{b}}$ & & .02 & .00 & $.10 * *$ \\
\hline
\end{tabular}

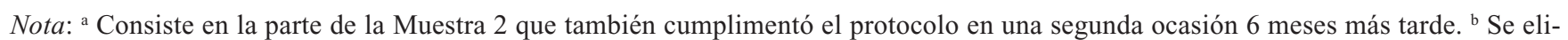
minaron los ítems del CSV referidos a salud propia (enfermedades). CD = Cuestionario de Depresión; EA = Escala de Ansiedad; PANAS-AN $=$ afecto negativo; PANAS-AP $=$ afecto positivo. ${ }^{*} p<0.05 ; * *<<0.01 ; * * *<0.001$.

rior. Por lo demás, los resultados son prácticamente idénticos a los obtenidos con el CSV completo. De nuevo constatamos que la varianza total explicada por el modelo de regresión es notablemente menor cuando se trata de predecir las enfermedades que cuando se refiere a la sintomatología psicológica (32-37\% vs. 7\%).

\section{Discusión}

La finalidad de este estudio fue proporcionar evidencia sobre la validación del CSV. En este sentido, hemos pretendido obtener información empírica sobre (a) la prevalencia y gravedad de los sucesos vitales, (b) la estructura factorial del cuestionario, (c) datos normativos (medias, desviaciones típicas, y puntos de corte basados en percentiles), y (d) la fiabilidad y la validez.

Respecto al primer objetivo hemos constatado, como cabría esperar, una notable dispersión en la frecuencia con que ocurren los diversos sucesos vitales. Aparte de los sucesos especialmente normativos por tratarse de una población académica (i.e., sucesos relacionados con los exámenes), o por ser muy normativos por naturaleza (p.ej., periodo de fiestas), la frecuencia de los sucesos varía entre los que parecen ser muy prevalentes (p.ej., problemas con los compañeros de trabajo; 62.3\%) y los que se dan con muy poca frecuencia (p.ej., muerte de un hijo; $0.3 \%$ ). Llama la atención que, entre los muy prevalentes (porcentaje $>20 \%$ ), se encuentran todos los sucesos vitales pertenecientes a la dimensión de trabajo, lo cual es consistente con el papel primordial que parece jugar el estrés asociado al trabajo (Esteras et al., 2014; Sandín, 2003). El resto de sucesos muy prevalentes se reparten entre las diversas dimensiones de estrés psicosocial, incluidas las relaciones de pareja, la salud, y la vivienda, fundamentalmente. Los sucesos de baja prevalencia (porcentaje $<5 \%$ ) se refieren a acontecimientos actualmente poco normativos en nuestra sociedad, entre los que se incluyen el embarazo, el nacimiento de un hijo, la complicación legal en una pelea, el aborto, o el alquiler de una vivienda. Finalmente, hemos constatado 
que algunos sucesos parecen ser significativamente más frecuentes en la mujer que en el hombre (quedarse sin trabajo, hospitalización de un familiar, retiro laboral, etc.), mientras que otros los padecen más los varones (problemas legales, problemas con compañeros y con superiores en el trabajo, problemas con el alcohol o drogas, etc.). Estas diferencias podrían explicarse, al menos parcialmente, en términos de diferencias de género en los patrones de conducta y roles psicosociales. No obstante, el perfil de frecuencia de los sucesos vitales más prevalentes es muy similar en ambos grupos.

Relacionado igualmente con el primer objetivo encontramos, también como cabría esperar, notables diferencias en el grado de estrés vinculado a cada suceso vital. Los sucesos percibidos como más estresantes tienen que ver principalmente con pérdidas mayores (p.ej., de un hijo, del cónyuge, de un familiar cercano, de un amigo íntimo, un divorcio, o la ruptura de un noviazgo o relación íntima). En el segundo nivel de estrés se ubican fundamentalmente sucesos relacionados con la salud (enfermedades, hospitalizaciones, lesiones físicas, etc.), problemas legales (problemas legales graves o leves, robo, etc.), vivienda, trabajo (pérdida de trabajo, problemas con superiores en el trabajo, etc.), y pérdidas económicas. Aunque ciertos sucesos parecen generar inevitablemente un nivel elevado de estrés, éste puede variar por las propias características personales, así como también por los propios cambios sociales. Por ejemplo, Holmes y Rahe (1967) asignaron al matrimonio 50 unidades de cambio vital (UCV), situándolo el puesto de séptimo suceso más estresante entre su lista de 43 eventos. En cambio, en posteriores revisiones de esta escala su posición (rango) bajó a los puestos 10 (1978) y 19 (1994), mientras que otros sucesos como el despido laboral, los problemas de salud de familiares, una hipoteca, un problema de negocios, o la muerte de un amigo íntimo, incrementaron significativamente el valor de UCV (Sandín y Chorot, 2008). A este respecto, es importante tener en cuenta que el CSV es sensible tanto a los posibles cambios sociales como a las propias diferencias individuales, razón por la cual es preferible la evaluación del impacto percibido por los individuos a la asignación externa de UCV. Por ejemplo, el suceso vital «divorcio o separación» aparece en las diversas revisiones del grupo de Holmes como un suceso con máximos valores de UCV. Sin embargo, en el presente estudio este evento fue experimentado por 93 personas, siendo valorado como nada o poco estresante por 16 personas, y como bastante estresante por 23 participantes; únicamente el resto $(58 \%)$ lo asoció a mucho o muchísimo estrés.

El segundo objetivo del estudio tenía como finalidad examinar la estructura factorial del CSV. Nuestros resul- tados sugieren una estructura de 7 factores aislados a partir de una población universitaria heterogénea (tanto en edad, como en otras variables sociodemográficas como la procedencia geográfica, el nivel económico, el estado civil, el trabajo, etc.). Esta estructura fue transvalidada a través de análisis factorial confirmatorio sobre la base de una muestra independiente, obtenida 3 años más tarde, perteneciente a una población similar. Los factores representan las 7 dimensiones psicosociales siguientes: trabajo, relaciones de pareja, vivienda/legal/social, pérdida laboral y/o de ingresos, salud de familiares, salud propia, y familia/hijos. Estas dimensiones son coherentes y relevantes desde el punto de vista del estrés psicosocial (Sandín, 2003, 2008). En general las dimensiones son bastante específicas, excepto la dimensión vivienda/ legal/social que implica al menos tres áreas conceptualmente diferentes aunque estrictamente interrelacionadas. Estas 7 dimensiones posibilitan configurar 7 escalas de estrés psicosocial mediante 42 ítems del cuestionario, siendo agrupados los restantes ítems en una escala residual que denominamos «miscelánea» (véase el Anexo I). Las correlaciones entre los factores (y/o entre las escalas) varían entre bajas y moderadas-bajas, lo cual sugiere la adecuación de poder utilizar las escalas de forma separada, aparte de utilizar la puntuación de estrés psicosocial total (basada en el CSV total o en el CSV-42).

Mediante el tercer objetivo deseábamos obtener datos normativos del cuestionario. A este respecto, hemos constatado información relevante $(M \mathrm{~s}, D T \mathrm{~s}$, diferencias entre varones y mujeres, y puntos de corte basados en percentiles) relacionada tanto con el CSV completo como con el CSV-42 (Tablas 6 y 7). En general se evidencia que los niveles de estrés correspondientes a las distintas áreas, al igual que al ámbito psicosocial global evaluado por el cuestionario, no parecen ser particularmente elevados, lo cual es coherente al tratarse de una población no clínica. Debe tenerse en cuenta, por otra parte, que estos valores se obtuvieron asignando valor cero a los sucesos no experimentados por el individuo, por lo cual una persona ha podido experimentar un suceso con mucho nivel de estrés pero puntuando globalmente de forma similar a otro individuo que haya experimentado varios sucesos con nivel bajo de estrés. Esta es una razón por la que la mera frecuencia de sucesos vitales no suele ser un buen indicador del estrés. Respecto a las comparaciones entre mujeres y varones, encontramos diferencias significativas en las puntuaciones totales de ambas formas del cuestionario, así como también en algunas escalas (relaciones de pareja, pérdida laboral y/o de ingresos, y problemas de salud familiar), lo cual es consistente con la idea de que, en general, la mujer suele ser más vulnerable al estrés que el hombre (Sandín, 2001). 
Finalmente, pretendíamos proporcionar datos sobre la fiabilidad y validez del CSV. La fiabilidad se ha estudiado centrándonos en la consistencia interna del cuestionario y de las escalas. Los coeficientes alfa indican que tal consistencia es elevada. Así mismo, las correlaciones corregidas ítem-escala sugieren aceptables niveles de consistencia. Tales resultados garantizan también la consistencia interna de las siete escalas que configuran el cuestionario CSV-42.

Los resultados basados en las correlaciones entre las variables del cuestionario y las variables psicopatológicas y de personalidad proporcionan evidencia sobre la validez convergente y discriminante del CSV. Como esperábamos, el CSV correlaciona de forma moderada-baja y significativa con las variables psicopatológicas (síntomas psicopatológicos, ansiedad, y depresión) y el afecto negativo, y no correlaciona con el afecto positivo. Estos resultados son consistentes con la evidencia de la literatura, que sugiere correlaciones entre moderadas y moderas-bajas entre el estrés psicosocial percibido y la sintomatología psiquiátrica y emocional (Clements y Turpin, 1996; Hammen, 2005; Phillips et al., 2015; Sandín, 2008; Sandín y Chorot, 1993; Sandín et al., 2004).

Las correlaciones obtenidas entre las variables de estrés psicosocial percibido y la ocurrencia posterior de enfermedades (incluye enfermedades graves o leves, visitas al médico y hospitalizaciones) son significativas pero más bajas que las correlaciones obtenidas con las variables psicopatológicas. Apreciamos, así mismo, que la ocurrencia de enfermedades correlaciona de forma similar con el CSV total que con el CSV-42, lo cual en cierto modo nos sorprende ya que algunos ítems relevantes (p.ej., generadores de alto nivel de estrés) no están incluidos en la forma reducida, si bien tales ítems suelen tener muy baja frecuencia. Estos resultados, no obstante, deben ser contrastados con nuevas investigaciones basadas en el CSV. Respecto a las correlaciones de enfermedades con las dimensiones psicosociales, todas se acercan al valor de .10. Aunque se trata de correlaciones bajas, todas son estadísticamente significativas y merecen ser tenidas en consideración. En general, estos datos son consistentes con la hipótesis sugerida por algunos autores (p.ej., Xing-jia y Vailant, 1996) de que el estrés psicosocial predice mejor las alteraciones de la salud psíquica que las de la salud física.

Finalmente, con el presente estudio proporcionamos evidencia relevante sobre la validez predictiva del CSV. Los resultados obtenidos a partir de los análisis de regresión indican que el estrés psicosocial vivido durante el último año (estrés psicosocial percibido) predice de forma significativa la sintomatología DSM-5, la ansiedad y la depresión evaluados seis meses más tarde por los par- ticipantes (síntomas referidos a las últimas semanas, autoinformados seis meses después de cumplimentar el CSV). Un aspecto importante a destacar consiste en que la capacidad predictiva del CSV se mantiene tras controlar el efecto de variables relevantes, incluyendo el género, la edad, el afecto negativo y el afecto positivo. El porcentaje de varianza explicada por todas estas variables es importante, siendo ligeramente más elevada al referirse a la ansiedad (hasta un 38\%). Los resultados obtenidos utilizando las puntuaciones en estrés basadas en el CSV-42 son bastante similares a las basadas en el CSV total (véanse las Tablas 9 y 10). Partiendo de los valores de beta, cabría afirmar que el estrés resulta ser el predictor más relevante tras el afecto negativo, variable ésta que suele asumirse como variable primaria de predisposición psicopatológica general (Sandín, 1990; Sandín y Chorot, 2009). El afecto positivo predice (inversamente) de forma importante el nivel de depresión, lo cual está en línea con la abundante evidencia que apoya el modelo tripartito sobre la afectividad, la ansiedad y la depresión (Joiner et al., 1998; Sandín y Chorot, 2009), en el cual se establece una conexión entre la depresión y el bajo afecto positivo.

En contraste con estos resultados, el porcentaje de varianza explicada en la regresión por las variables independientes respecto a la ocurrencia de enfermedades es notablemente inferior (7/8\%). Esto indica que el estrés psicosocial percibido basado en sucesos vitales recientes, al igual que las variables de personalidad, predice en el tiempo la ocurrencia de la enfermedad, aunque de forma más limitada que los problemas de salud de tipo psicológico. Esta conclusión, que ya había sido sugerida previamente por Xing-jia y Vailant (1996), parece derivarse de los resultados del presente estudio. Observamos por otra parte, una variación en cuanto a la relevancia de la edad y el género pues, mientras que la primera parece jugar un cierto papel en la predicción de los síntomas psicopatológicos y la ansiedad (la edad predice menor grado de sintomatología), la segunda lo es fundamentalmente en relación con las enfermedades (el género femenino predice mayor puntuación en enfermedades). El porcentaje de varianza explicada en enfermedades es significativo y, aunque es bajo, es importante tener en cuenta que la puntuación en enfermedades corresponde a un periodo de tiempo posterior al periodo sobre el que se evaluó el estrés psicosocial percibido (i.e., los 6 meses posteriores). Es posible que la puntuación en enfermedades evaluada en el Tiempo 2 (referida a los 6 meses previos) pueda depender en parte del nivel de enfermedades evaluado en el Tiempo 1 (referido a los 12 meses previos) $(r=.59)$, pero es igualmente cierto que el nivel de enfermedades en el Tiempo 1 puede 
estar asociado al nivel de estrés referido al mismo periodo (12 meses), razón por la cual en este contexto no consideramos apropiado incluir la variable enfermedades en Tiempo 1 como variable a controlar en los análisis de regresión.

Los resultados basados en los análisis de regresión, en los cuales se ha controlado el efecto de la afectividad negativa, sugieren que los efectos del estrés psicosocial sobre la psicopatología y la enfermedad son independientes del efecto que ejerce la afectividad negativa sobre dichas variables. Nuestro grupo también ha demostrado que esto mismo ocurre en contextos en los que se evalúa el estrés diario, en lugar del estrés reciente (Santed et al., 2000), aunque en la literatura se ha generado cierta polémica sobre la validez de la evaluación del estrés psicosocial en términos de estrés percibido por la amenaza impuesta por el efecto de la afectividad negativa. En esencia se parte de que los individuos con elevado afecto negativo tienden a percibir los sucesos vitales como más negativos, confundiéndose ambos posibles efectos (el de los sucesos vitales y el del afecto negativo); para una revisión y análisis de esta problemática, véase Santed, Sandín, Chorot, Olmedo, y García-Campayo, 2003).

Aunque el presente estudio combina aspectos transversales y aspectos longitudinales y proporciona información relevante sobre la estructura y validación del CSV, presenta también algunas limitaciones. Una primera cuestión es que únicamente se ha evaluado el impacto personal de los sucesos vitales (grado de estrés o malestar), quedando fuera de la evaluación las dos características de la demanda psicosocial que permite evaluar el cuestionario, i.e., la valencia (positivo vs. negativo) y la predecibilidad (esperado vs. inesperado). Por ejemplo, ciertos sucesos vitales pueden ser valorados como positivos (p.ej., «éxito personal importante»), lo cual podría afectar de algún modo a la salud. Un segundo problema es que en el presente estudio no se evaluaron otros tipos de estresores psicosociales (p.ej., estrés diario), y como sabemos los diferentes tipos de sucesos pueden estar interrelacionados entre sí (Sandín, 2003). Lógicamente, la consideración de otras fuentes de estrés habría incrementado la capacidad predictiva del estrés psicosocial sobre la salud (Sandín et al., 2002). En tercer lugar, en este estudio hemos utilizado un tipo de población de alto nivel cultural (estudiantes universitarios). Aunque como hemos indicado no se trata de un grupo homogéneo socio-demográficamente hablando (las muestras no corresponden a la población típica de estudiantes universitarios de las universidades presenciales), sería deseable ampliar la validación del cuestionario empleando otros tipos de población. Finalmente, sería aconsejable utilizar el cuestionario en estudios longitudinales que posibilitasen establecer claras relaciones causa- les entre el estrés y los diferentes tipos de problemas y trastornos relacionados con la salud mental y física.

A modo de conclusión y recomendación final, el CSV ha demostrado ser válido y relativamente fiable (la fiabilidad test-retest no ha podido calcularse ya que los dos momentos en que se aplicó el CSV debían haber hecho referencia al mismo periodo de tiempo). No obstante, existe evidencia de que los sucesos vitales mayores suelen recordarse bastante bien durante un periodo de un año, e incluso más (Sandín, 2008). Por otra parte, hemos aislado varias dimensiones psicosociales relevantes para el estrés psicosocial, a pesar de que los sucesos pueden relacionarse entre sí, y los de un área puede influir sobre los sucesos de otra (p.ej., un divorcio puede influir en las relaciones familia/hijos). Los resultados de los análisis factoriales y de frecuencia nos han llevado a eliminar el ítem 60 («alquilar una vivienda»), integrando su contenido en el nuevo ítem 26 («cambio de casa o tener que buscar o alquilar nueva vivienda»). Así mismo, se suprimen los contenidos de los ítems 32 y 35 , que han resultado ser irrelevantes, por los nuevos contenidos siguientes que fueron autoinformados en el presente estudio: «sufrir agresiones físicas de su pareja» (ítem 32) y «ser denunciado/a por malos tratos por su pareja» (ítem 35). El cuestionario podrá utilizarse con la totalidad de los 59 ítems, o bien aplicando únicamente la versión de 44 ítems (estos dos nuevos ítems se han integrado racionalmente en la dimensión de relaciones de pareja, pasando el cuestionario abreviado a sumar 44 ítems) (véase el Anexo I). Aparte de estas dimensiones aisladas empíricamente, es factible elaborar racionalmente otros tipos de dimensiones en función de los intereses de la investigación, incluyendo las de dependientes-independientes, pérdidas, amenaza/peligro/daño, salud, etc. (Sandín et al., 2002, 2003).

\section{Conflictos de intereses}

Los autores declaran que no existen conflictos de intereses

\section{Referencias}

American Psychiatric Association (2013a). Diagnostic and statistical manual of mental disorders ( $5^{\text {th }}$ ed., DSM-5). Washington, DC: Author.

American Psychiatric Association (2013b). DSM-5 Self-Rated Level 1 Cross-Cutting Symptom MeasureAdult. Online Assessment Measures. https://www.psychiatry.org/psychiatrists/ practice/dsm/educational-resources/assessment-measures.

Ballester-Arnal, R., Gómez-Martínez, S., Gil-Juliá, B., Ferrándiz-Sellés, M. D., y Collado-Boira, E. J. (2016). Burnout y factores estresantes en profesionales sanitarios de las unida- 
des de cuidados intensivos. Revista de Psicopatología y Psicología Clínica, 21, 129-136.

Berntson, J., Patel, J. S., \& Stewart, J. C. (2017). Number of recent stressful life events and incident cardiovascular disease: Moderation by lifetime depressive disorder. Journal of Psychosomatic Research, 99, 149-154.

Brown, T. A. (2006). Confirmatory factor analysis for applied research. New York: Guilford Press.

Byrne, B. M. (2006). Structural equation modelling with EQS ( $2^{\text {nd }}$ ed.). New York: Routledge.

Catell, R. B. (1966). The scree test for the number of factors. Multivariate Behavioral Research, 3, 245-276.

Clements, K., \& Turpin, G. (1996). The life events scale for students: Validation for use with British samples. Personality and Individual Differences, 20, 747-751.

Chorot, P., \& Sandín, B. (1994). Life events and stress reactivity as predictors of cancer, coronary heart disease and anxiety disorders. International Journal of Psychosomatics, 41, 34-40.

Echeburúa, E. (2009). Trastorno de estrés postraumático. En A. Belloch, B. Sandín y F. Ramos (Eds.), Manual de psicopatología (Vol.2, pp. 137-148).

Esteras, J., Chorot, P., y Sandín, B. (2014). Predicción de los niveles de burnout en los docentes: Factores protectores y de vulnerabilidad. Revista de Psicopatología y Psicología Clínica, 19, 79-92.

Hammen, C. (2005). Stress and depression. Annual Review of Clinical Psychology, 1, 293-319.

Heikkinen, S., Miettinen, J., Pukkala, E., Koskenvuo, M., Malila, N., \& Pitkäniemi, J. (2017). Impact of major life events on breast-cancer-specific mortality: A case fatality study on 8000 breast cancer patients. Cancer Epidemiology, 48, 62-69.

Holmes, T. H., \& David, E. M. (Eds.) (1989). Life change, life events, and illness. New York: Praeger

Holmes, T. H., \& Rahe, R. H. (1967). The Social Readjusment Rating Scale. Journal of Psychosomatic Research, 11, 213218.

Horn, J. L. (1965). A rationale and test for the number of factors in factor analysis. Psychometrika, 30, 179-185.

Hu, L., \& Bentler, P. (1999). Cutoff criteria for fit indexes in covariance structure analysis: Conventional criteria versus new alternatives. Structural Equations Modeling, 6, 1-55.

Kokoulina, E., \& Fernández, R. (2014). Maltrato físico y emocional en la infancia y conducta suicida en el adulto. Revista de Psicopatología y Psicología Clínica, 19, 93-103.

Krenek, M., \& Maisto, S. A. (2013). Life events and treatment outcomes among individuals with substance use disorders: A narrative review. Clinical Psychology Review, 33, 470-483.

Liu, R. T., \& Miller, I. (2014). Life events and suicidal ideation and behavior: A systematic review. Clinical Psychology Review, 34(3), 181-192.

Mansueto, G., \& Faravelli, C. (2017). Recent life events and psychosis: The role of childhood adversities. Psychiatry Research, 256, 111-117.

Miller, T. W. (1997). Clinical disorders and stressful life events. Madison: International Universities Press.

Phillips, A. C., Carroll, D., \& Der, G. (2015). Negative life events and symptoms of depression and anxiety: stress causation and/or stress generation. Anxiety, Stress \& Coping, 28, 357371.
Porcelli, B., Pozza, A., Bizzaro, N., Fagiolini, A., Costantini, M. C., Terzuoli, L., \& Ferretti, F. (2016). Association between stressful life events and autoimmune diseases: A systematic review and meta-analysis of retrospective case-control studies. AutoimmunityReviews, 15, 325-334.

Sandín, B. (1989). Estrés, coping y alteraciones psicofisiológicas. En B. Sandín y J. Bermúdez (Eds.), Procesos emocionales y salud (pp. 45-72). Madrid: UNED.

Sandín, B. (1990). Factores de predisposición en los trastornos de ansiedad. Revista de Psicología General y Aplicada, 43, 343-351.

Sandín, B. (1993). Estrés y salud: Factores que intervienen en la relación entre el estrés y la enfermedad física. En J. Buendía (Ed.), Estrés y psicopatología (pp. 148-180). Madrid: Pirámide.

Sandín, B. (2001). Estrés, hormonas y psicopatología. Madrid: Klinik.

Sandín, B. (2003). El estrés: Un análisis basado en el papel de los factores sociales. Revista Internacional de Psicología Clínica $y$ de la Salud, 3, 141-157.

Sandín, B. (2009). El estrés. En A. Belloch, B. Sandín y F. Ramos (Eds.). Manual de psicopatología, Vol. 2 (ed. rev., pp. 3-42). Madrid: McGraw-Hill.

Sandín, B. (Ed.) (2008). El estrés psicosocial: Conceptos y consecuencias clínicas (2 $2^{\mathrm{a}}$ ed.). Madrid: Klinik.

Sandín, B., y Chorot, P. (1987). Cuestionario de Sucesos Vitales (CSV). Madrid: Universidad Nacional de Educación a Distancia (no publicado).

Sandín, B., \& Chorot, P. (1993). Stress and anxiety: Diagnosis validity of anxiety disorders according to life events stress, ways of coping and physical symptoms. Psiquis, 14, 178-184.

Sandín, B., y Chorot, P. (1996). Evaluación del estrés psicosocial. En G. Buela-Casal, V. Caballo y J.C. Sierra (Eds.), Evaluación en psicología clínica (pp. 619-656). Madrid: Siglo XXI.

Sandín, B., y Chorot, P. (2008). Evaluación de los sucesos vitales. En B. Sandín (Ed.), El estrés psicosocial: Conceptos y consecuencias clínicas ( $2^{\mathrm{a}}$ edición, pp. 65-83). Madrid: Klinik.

Sandín, B., y Chorot, P. (2009). Concepto y categorización de los trastornos de ansiedad. En A. Belloch, B. Sandín y F. Ramos (Eds.), Manual de psicopatología, Vol. 2 (ed. rev., 43-63). Madrid: McGraw-Hill.

Sandín, B., Chorot, P., Lostao, L., Joiner, T.E., Santed, M.A., y Valiente, R.M. (1999). Escalas PANAS de afecto positivo y negativo: Validación factorial y convergencia transcultural. Psicothema, 11, 37-51.

Sandín, B., Chorot, P., Santed, M.A., y Jiménez, P. (2009). Trastornos psicosomáticos. En A. Belloch, B. Sandín y F. Ramos (Eds.), Manual de psicopatología, Vol. 2 (ed. rev., 315-367). Madrid: McGraw-Hill.

Sandín, B., Chorot, P., Santed, M.A., y Valiente, R.M. (2002). Estrés y salud: Relación de los sucesos vitales y el estrés diario con la sintomatología somática y la enfermedad. Ansiedad y Estrés, 8, 73-87.

Sandín, B., Chorot, P., Santed, M.A., \& Valiente, R.M. (2004). Differences in negative life events between patients with anxiety disorders, depression and hypochondriasis. Anxiety, Stress and Coping, 17, 37-47.

Sandín, B., Chorot, P., Santed, M.A., Valiente, R., \& Joiner, T.E. (1998). Negative life events and adolescent suicidal behavior: A critical analysis from the stress process perspective. Journal of Adolescence, 21, 415-426. 
Sandín, B., Chorot, P., y Valiente, R. M. (2011). Escala de Ansiedad (EA). Madrid: Universidad Nacional de Educación a Distancia (no publicado).

Sandín, B., Chorot, P., y Valiente, R. M. (2013). Versión española del DSM-5 Self-Rated Level 1 Cross-Cutting Symptom Measure-Adult. Madrid: Universidad Nacional de Educación a Distancia (no publicado).

Sandín, B., Rodero, B., Santed, M.A., y García-Campayo, J. (2006). Sucesos vitales estresantes y trastorno de pánico: Relación con la ocurrencia del trastorno de pánico, la gravedad clínica y la agorafobia. Revista de Psicopatología y Psicología Clínica, 11, 179-190.

Sandín, B., y Valiente, R. M. (1998). Cuestionario de Depresión (CD). Madrid: Universidad Nacional de Educación a Distancia (no publicado).

Sandín, B., Valiente, R.M., y Chorot, P. (2008). Instrumentos para la evaluación del estrés psicosocial. En B. Sandín (Ed.), El estrés psicosocial: Conceptos y consecuencias clínicas $\left(2^{\mathrm{a}}\right.$ ed., pp. 275-358). Madrid: Klinik.

Santandreu, M., \& Ferrer, V. A. (2014). Análisis de la emotividad negativa en mujeres víctimas de violencia de pareja: La culpa y la ira. Revista de Psicopatología y Psicología Clínica, 19, 129-140.

Santed, M.A., Sandín, B., y Chorot, P. (1996). Cuestionario de Estrés Diario (CED): Validez de constructo y el problema de la confusión de medidas. Boletín de Psicología, 51, 45-70.

Santed, M.A., Sandín, B., y Chorot, P. (1998). El estrés diario desde el marco del modelo transaccional: Cuestiones conceptuales y metodológicas. En J. Buendía (Ed.), Estrés laboral y salud (pp. 21-49). Madrid: Biblioteca Nueva.

Santed, M.A., Sandín, B., Chorot, P., y Olmedo, M. (2000). Predicción de la sintomatología somática a partir del estrés diario: Un estudio prospectivo controlando el efecto del neuroticismo. Revista de Psicopatología y Psicología Clínica, 5, 165-178.

Santed, M.A., Sandín, B., Chorot, P., y Olmedo, M. (2000). Predicción de la sintomatología somática a partir del estrés diario y de los síntomas previos: Un estudio prospectivo. Ansiedad y Estrés, 6, 317-329.
Santed, M.A., Sandín, B., Chorot, P., Olmedo, M., \& García-Campayo, J. (2003). The role of negative and positive affectivity on perceived stress-subjective health relationships. Acta Neuropsychiatrica, 15, 199-216.

Tabachnik, B.G., \& Fidell, L.S. (2007). Using multivariate statistics $\left(5^{\text {th }}\right.$ ed.). Boston, MA: Pearson.

Tobón, S., Sandín, B., y Vinaccia, S. (2006). Trastornos gastrointestinales: Psicopatología y tratamientos psicológicos. Madrid: Klinik.

Tobón, S., Vinaccia, S., y Sandín, B. (2003). Implicación del estrés psicosocial y los factores psicológicos en la dispepsia funcional. Anales de Psicología, 19, 223-234.

Tobón, S., Vinaccia, S., y Sandín, B. (2004). Modelo procesual del estrés en la dispepsia funcional: Implicaciones para la evaluación y el tratamiento. Revista de Psicopatología y Psicología Clínica, 9, 81-98.

Valiente, R.M., Sandín, B., Chorot, P., Santed, M.A., y González de Rivera, J.L. (1996). Sucesos vitales mayores y estrés: Efectos del cambio por migración asociados a manifestaciones psicopatológicas. Psiquis, 17, 211-230.

Vinaccia, S., Tobón, S., Martínez, F., y Sandín, B. (2001). Estrés y úlcera péptica duodenal en sujetos infectados con helicobacter pylori: Un estudio controlado. Revista Internacional de Psicología Clínica y de la Salud, 1, 317-331.

Vinaccia, S., Tobón, S., Sandín, B., y Martínez, F. (2001). Estrés psicosocial y úlcera péptica duodenal: Una perspectiva bio-psico-social. Revista Latinoamericana de Psicología, 33, 117-130.

Watson, D., Clark, L.A., \& Tellegen, A. (1988). Development and validation of brief measures of positive and negative affect: The PANAS scales. Journal of Personality and Social Psychology, 54, 1063-1070.

Xavier, A., Cunha, M., \& Pinto Gouveia, J. (2015). Deliberate self-harm in adolescence: The impact of childhood experiences, negative affect and fears of compassion. Revista de Psicopatología y Psicología Clínica, 20, 41-49.

Xing-jia, C., y Vailant, G. E. (1996). Antecedents and consequences of negative life events in adulthood: A longitudinal study. American Journal of Psychiatry, 153, 21-26. 
Anexo I.

Cuestionario de Sucesos Vitales (CSV). Formato de aplicación y normas para la corrección de la prueba

Nombre

Edad

Ocupación.

Fecha

Instrucciones: A continuación encontrará una lista de sucesos vitales que suelen ocurrir a la gente. No todas las personas experimentan los mismos sucesos; cada persona vivencia los sucesos particulares que le ocurren. Lea detenidamente la lista de sucesos. Cuando se encuentre con sucesos que a usted NO le han ocurrido en el último año no señale nada. Sin embargo, cuando se encuentre ante sucesos que SÍ le han ocurrido durante el último año, párese en cada uno y conteste a los tres apartados que se piden rodeando con un círculo el lugar apropiado de acuerdo con su experiencia personal. Estos tres apartados son:

1. Grado de estrés, tensión o malestar producido por el suceso: Rodee con un círculo el número que mejor se ajuste al grado de estrés, tensión o malestar que le ha producido o está produciendo el suceso en cuestión, teniendo en cuenta que:

1 Nada/muy poco 2 Bastante 3 Mucho 4 Muchísimo

2. Cómo evaluó el suceso (positivo o negativo): Rodee la $\mathbf{P}$ si cree que el suceso fue para usted fue algo positivo, o la $\mathbf{N}$ si lo evaluó como algo negativo.

3. Hasta qué punto sabía que iba a ocurrir el suceso: Rodee la E si se trata de un suceso que usted ya esperaba, o la I si se trata de un suceso inesperado.

\begin{tabular}{|c|c|c|c|c|c|c|c|c|}
\hline \multirow{2}{*}{ 1. Quedarse sin trabajo } & \multicolumn{4}{|c|}{$\begin{array}{l}\text { Grado de } \\
\text { estrés }\end{array}$} & \multicolumn{2}{|c|}{$\begin{array}{l}\text { Positivo (P) } \\
\text { Negativo (N) }\end{array}$} & \multicolumn{2}{|c|}{$\begin{array}{r}\text { Esperado (E) } \\
\text { Inesperado (I) }\end{array}$} \\
\hline & 1 & 2 & 3 & 4 & $\mathrm{P}$ & $\mathrm{N}$ & $\mathrm{E}$ & I \\
\hline 2. Enfermedad prolongada que requiere tratamiento & 1 & 2 & 3 & 4 & $\mathrm{P}$ & $\mathrm{N}$ & $\mathrm{E}$ & I \\
\hline 3. Problemas con colegas o compañeros de trabajo & 1 & 2 & 3 & 4 & $\mathrm{P}$ & $\mathrm{N}$ & $\mathrm{E}$ & I \\
\hline 4. Retiro laboral (jubilarse) & 1 & 2 & 3 & 4 & $\mathrm{P}$ & $\mathrm{N}$ & $\mathrm{E}$ & I \\
\hline 5. Matrimonio & 1 & 2 & 3 & 4 & $\mathrm{P}$ & $\mathrm{N}$ & $\mathrm{E}$ & I \\
\hline $\begin{array}{l}\text { 6. Problemas con los hijos (no poder verlos por separación, algún problema } \\
\text { grave en el colegio, etc.) }\end{array}$ & 1 & 2 & 3 & 4 & $\mathrm{P}$ & $\mathrm{N}$ & $\mathrm{E}$ & I \\
\hline 7. Nacimiento de un hijo & 1 & 2 & 3 & 4 & $\mathrm{P}$ & $\mathrm{N}$ & $\mathrm{E}$ & I \\
\hline 8. Divorcio o separación & 1 & 2 & 3 & 4 & $\mathrm{P}$ & $\mathrm{N}$ & $\mathrm{E}$ & I \\
\hline 9. Problema legal grave (con o sin cárcel) & 1 & 2 & 3 & 4 & $\mathrm{P}$ & $\mathrm{N}$ & $\mathrm{E}$ & I \\
\hline 10. Exámenes importantes & 1 & 2 & 3 & 4 & $\mathrm{P}$ & $\mathrm{N}$ & $\mathrm{E}$ & I \\
\hline 11. Reducción sustancial en los ingresos & 1 & 2 & 3 & 4 & $\mathrm{P}$ & $\mathrm{N}$ & $\mathrm{E}$ & I \\
\hline 12. Reformas en la casa & 1 & 2 & 3 & 4 & $\mathrm{P}$ & $\mathrm{N}$ & $\mathrm{E}$ & I \\
\hline 13. Muerte del cónyuge & 1 & 2 & 3 & 4 & $\mathrm{P}$ & $\mathrm{N}$ & $\mathrm{E}$ & I \\
\hline 14. Problemas legales relacionados con el alcohol o las drogas & 1 & 2 & 3 & 4 & $\mathrm{P}$ & $\mathrm{N}$ & $\mathrm{E}$ & I \\
\hline 15. Ascenso en el trabajo & 1 & 2 & 3 & 4 & $\mathrm{P}$ & $\mathrm{N}$ & $\mathrm{E}$ & I \\
\hline 16. Sufrir malos tratos de su pareja & 1 & 2 & 3 & 4 & $\mathrm{P}$ & $\mathrm{N}$ & $\mathrm{E}$ & I \\
\hline 17. Comprar una casa & 1 & 2 & 3 & 4 & $\mathrm{P}$ & $\mathrm{N}$ & $\mathrm{E}$ & $\mathrm{I}$ \\
\hline 18. Cambio de lugar de trabajo & 1 & 2 & 3 & 4 & $\mathrm{P}$ & $\mathrm{N}$ & $\mathrm{E}$ & I \\
\hline 19. Ruptura de noviazgo o relación similar & 1 & 2 & 3 & 4 & $\mathrm{P}$ & $\mathrm{N}$ & $\mathrm{E}$ & I \\
\hline 20. Muerte de un amigo íntimo & 1 & 2 & 3 & 4 & $\mathrm{P}$ & $\mathrm{N}$ & $\mathrm{E}$ & I \\
\hline 21. Muerte de un hijo & 1 & 2 & 3 & 4 & $\mathrm{P}$ & $\mathrm{N}$ & $\mathrm{E}$ & I \\
\hline 22. Relaciones sexuales fuera del matrimonio & 1 & 2 & 3 & 4 & $\mathrm{P}$ & $\mathrm{N}$ & $\mathrm{E}$ & I \\
\hline 23. Hijos lejos del hogar & 1 & 2 & 3 & 4 & $\mathrm{P}$ & $\mathrm{N}$ & $\mathrm{E}$ & I \\
\hline 24. Complicación legal en una pelea & 1 & 2 & 3 & 4 & $\mathrm{P}$ & $\mathrm{N}$ & $\mathrm{E}$ & $\mathrm{I}$ \\
\hline 25. Problema legal menor (multa, etc.) & 1 & 2 & 3 & 4 & $\mathrm{P}$ & $\mathrm{N}$ & $\mathrm{E}$ & I \\
\hline 26. Cambio de casa o tener que buscar o alquilar una nueva vivienda & 1 & 2 & 3 & 4 & $\mathrm{P}$ & $\mathrm{N}$ & $\mathrm{E}$ & I \\
\hline 27. Problemas con vecinos o con familiares que no viven en la casa familiar & 1 & 2 & 3 & 4 & $\mathrm{P}$ & $\mathrm{N}$ & E & $\mathrm{I}$ \\
\hline 28. Abortar & 1 & 2 & 3 & 4 & $\mathrm{P}$ & $\mathrm{N}$ & $\mathrm{E}$ & I \\
\hline
\end{tabular}


29. Aborto de su mujer o pareja

\begin{tabular}{|c|c|c|c|c|}
\hline 3 & 4 & $\mathrm{P}$ & $\mathrm{N}$ & $\mathrm{E}$ \\
\hline 3 & 4 & $\mathrm{P}$ & $\mathrm{N}$ & $\mathrm{E}$ \\
\hline 3 & 4 & $\mathrm{P}$ & $\mathrm{N}$ & $\mathrm{E}$ \\
\hline 3 & 4 & $\mathrm{P}$ & $\mathrm{N}$ & $\mathrm{E}$ \\
\hline 3 & 4 & $\mathrm{P}$ & $\mathrm{N}$ & $\mathrm{E}$ \\
\hline 3 & 4 & $\mathrm{P}$ & $\mathrm{N}$ & $\mathrm{E}$ \\
\hline 3 & 4 & $\mathrm{P}$ & $\mathrm{N}$ & $\mathrm{E}$ \\
\hline 3 & 4 & $\mathrm{P}$ & $\mathrm{N}$ & $\mathrm{E}$ \\
\hline 3 & 4 & $\mathrm{P}$ & $\mathrm{N}$ & $\mathrm{E}$ \\
\hline 3 & 4 & $\mathrm{P}$ & $\mathrm{N}$ & $\mathrm{E}$ \\
\hline 3 & 4 & $\mathrm{P}$ & $\mathrm{N}$ & $\mathrm{E}$ \\
\hline 3 & 4 & $\mathrm{P}$ & $\mathrm{N}$ & $\mathrm{E}$ \\
\hline 3 & 4 & $\mathrm{P}$ & $\mathrm{N}$ & $\mathrm{E}$ \\
\hline 3 & 4 & $\mathrm{P}$ & $\mathrm{N}$ & $\mathrm{E}$ \\
\hline 3 & 4 & $\mathrm{P}$ & $\mathrm{N}$ & $\mathrm{E}$ \\
\hline 3 & 4 & $\mathrm{P}$ & $\mathrm{N}$ & $\mathrm{E}$ \\
\hline 3 & 4 & $\mathrm{P}$ & $\mathrm{N}$ & $\mathrm{E}$ \\
\hline 3 & 4 & $\mathrm{P}$ & $\mathrm{N}$ & $\mathrm{E}$ \\
\hline 3 & 4 & $\mathrm{P}$ & $\mathrm{N}$ & $\mathrm{E}$ \\
\hline 3 & 4 & $\mathrm{P}$ & $\mathrm{N}$ & $\mathrm{E}$ \\
\hline 3 & 4 & $\mathrm{P}$ & $\mathrm{N}$ & $\mathrm{E}$ \\
\hline 3 & 4 & $\mathrm{P}$ & $\mathrm{N}$ & $\mathrm{E}$ \\
\hline 3 & 4 & $\mathrm{P}$ & $\mathrm{N}$ & $\mathrm{E}$ \\
\hline 3 & 4 & $\mathrm{P}$ & $\mathrm{N}$ & $\mathrm{E}$ \\
\hline 3 & 4 & $\mathrm{P}$ & $\mathrm{N}$ & $\mathrm{E}$ \\
\hline 3 & 4 & $\mathrm{P}$ & $\mathrm{N}$ & $\mathrm{E}$ \\
\hline 3 & 4 & $\mathrm{P}$ & $\mathrm{N}$ & $\mathrm{E}$ \\
\hline 3 & 4 & $\mathrm{P}$ & $\mathrm{N}$ & $\mathrm{E}$ \\
\hline 3 & 4 & $\mathrm{P}$ & $\mathrm{N}$ & $\mathrm{E}$ \\
\hline 3 & 4 & $\mathrm{P}$ & $\mathrm{N}$ & $\mathrm{E}$ \\
\hline 3 & 4 & $\mathrm{P}$ & $\mathrm{N}$ & $\mathrm{E}$ \\
\hline
\end{tabular}

30. Nuevo empleo laboral

31. Muerte de algún familiar cercano (padre, hermano, etc.)

32. Sufrir agresiones físicas de su pareja

33. Préstamo o hipoteca de más de $100.000 €$

34. Sufrir robo

35. Ser denunciado/a por malos tratos por su pareja

36. Enfermedad o accidente que requiere guardar cama

37. Finalización de estudios

38. Accidente de tráfico

39. Repentino y serio deterioro de la audición o visión

40. Lesión física importante

41. Suspender exámenes

42. Quedarse embarazada

43. Enamorarse o iniciar una amistad íntima y profunda

44. Embarazo de su mujer o pareja

45. Enfermedad de un familiar

46. Ruptura de relaciones sexuales fuera del matrimonio

47. Cambio de horario o de las condiciones del trabajo actual

48. Vivir temporalmente fuera del hogar

49. Reconciliación matrimonial o de pareja

50. Separación de los padres

51. Fracaso en algún negocio importante

52. Hospitalización de algún familiar

53. Sufrir asalto o situación de violencia física

54. Pérdidas económicas importantes no asociadas al trabajo

55. Cambio de centro de estudios

56. Problemas con superiores en el trabajo

57. Éxito personal importante

58. El cónyuge comienza a trabajar fuera de casa

59. Discusión especialmente grave con su cónyuge o pareja

60. Indicar otros sucesos importantes no señalados

$\begin{array}{llllllll}1 & 2 & 3 & 4 & \mathrm{P} & \mathrm{N} & \mathrm{E} & \mathrm{I} \\ 1 & 2 & 3 & 4 & \mathrm{P} & \mathrm{N} & \mathrm{E} & \mathrm{I}\end{array}$

(C) 1987 B. Sandín y P. Chorot. Universidad Nacional de Educación a Distancia, Madrid.

Corrección de la prueba:

Pueden obtenerse las siguientes puntuaciones:

1) Número total de sucesos vitales (sumando los sucesos no marcados).

2) Grado de estrés percibido (sumando las puntuaciones marcadas para cada ítem).

3) Grado de estrés percibido según las diferentes subescalas:

Trabajo: 3, 15, 18, 30, 47, 56, 57.

Relaciones de pareja: 8, 16, 19, 22, 32, 35, 43, 46, 49, 59.

Vivienda/Legal/Social: 12, 14, 17, 25, 26, 27, 34, 48, 50, 53.

Salud familia: 31, 45, 52.

Salud propia: 2, 36, 38, 39, 40 .

Familia/Hijos: 5, 6, 23, 33.

Pérdida laboral/Ingresos: 1, 4, 11, 51, 54.

Miscelánea: 7, 9, 10, 13, 20, 21, 24, 28, 29, 37, 41, 42, 44, 55, 58.

Nota. Los Ítems 32 y 35 de la versión anterior han sido modificados, siendo actualmente asignados racionalmente a la dimensión de relaciones de pareja, por lo que el CSV-42 definido factorialmente en el presente estudio pasaría a tener 44 ítems (CSV-44). El CSV-44 incluye todos los ítems del CSV excepto los correspondientes a la dimensión de Miscelánea.

Adaptado de Sandín, B., Valiente, R.M., y Chorot, P. (2008). Instrumentos para la evaluación del estrés psicosocial. En B. Sandín (Ed.), El estrés psicosocial: Conceptos y consecuencias clínicas (2a ed., pp. 267-346). Madrid: Klinik. Reproducido con permiso. Utilícese esta referencia al citar el CSV. 